\title{
Effective action for gauge bosons
}

\author{
Jérémie Quevillon, ${ }^{*}$ Christopher Smith, ${ }^{\dagger}$ and Selim Touati ${ }^{\ddagger}$ \\ Laboratoire de Physique Subatomique et de Cosmologie, Université Grenoble-Alpes, \\ CNRS/IN2P3, Grenoble INP, 38000 Grenoble, France
}

(Received 27 October 2018; published 16 January 2019)

\begin{abstract}
By treating the vacuum as a medium, H. Euler and W. Heisenberg estimated the nonlinear interactions between photons well before the advent of quantum electrodynamics. In a modern language, their result is often presented as the archetype of an effective field theory (EFT). In this work, we develop a similar EFT for the gauge bosons of some generic gauge symmetry, valid for example for $S U(2), S U(3)$, various grand unified groups, or mixed $U(1) \otimes S U(N)$ and $S U(M) \otimes S U(N)$ gauge groups. Using the diagrammatic approach, we perform a detailed matching procedure which remains manifestly gauge invariant at all steps, but does not rely on the equations of motion hence is valid off shell. We provide explicit analytic expressions for the Wilson coefficients of the dimension four, six, and eight operators as induced by massive scalar, fermion, and vector fields in generic representations of the gauge group. These expressions rely on a careful analysis of the quartic Casimir invariants, for which we provide a review using conventions adapted to Feynman diagram calculations. Finally, our computations show that at one loop, some operators are redundant whatever the representation or spin of the particle being integrated out, reducing the apparent complexity of the operator basis that can be constructed solely based on symmetry arguments.
\end{abstract}

DOI: 10.1103/PhysRevD.99.013003

\section{INTRODUCTION}

In 1936, H. Euler and W. Heisenberg calculated the nonlinear interactions among photons for a constant Maxwell field, as induced by a spinor loop [1]. This has been an important step in the development of QED, and their result remains as the canonical example of an effective field theory (EFT). That is, the idea that at energies below some cutoff scale $\Lambda$, all the effects of the degrees of freedom more massive than $\Lambda$ can be encoded as new interactions among the fields remaining active below $\Lambda$. This concept is central to modern phenomenology. The Fermi theory of the weak interactions [2] and the chiral Lagrangian of pions [3] have played an important role in the development of the Standard Model $[4,5]$. The methodology has since been used to define many other frameworks to either simplify the problem at hand, or to parametrize possible New Physics effects, for example for neutrino [6], nuclear [7], flavor [8], electroweak [9,10], and Higgs physics [11], or more globally for the Standard Model (SMEFT) [12]. Few developments have

\footnotetext{
*jeremie.quevillon@1psc.in2p3.fr chsmith@lpsc.in2p3.fr

touati@1psc.in2p3.fr
}

Published by the American Physical Society under the terms of the Creative Commons Attribution 4.0 International license. Further distribution of this work must maintain attribution to the author(s) and the published article's title, journal citation, and DOI. Funded by SCOAP ${ }^{3}$. also been done regarding EFTs for dark matter [13], inflation [14] and cosmology [15].

The purpose of the present paper is to generalize the Euler-Heisenberg $(\mathrm{EH})$ result for photons to the gauge bosons of an arbitrary gauge group, with their effective interactions induced by loops of heavy fields in generic representations and of spin $0,1 / 2$, or 1 . Let us thus first recall a few facts about the EH Lagrangian, and some of its applications.

In QED, for energies below the electron mass $m_{e}$, the photons can interact between themselves only indirectly via virtual loops of electron-positron extracted from the vacuum. These interactions are suppressed by inverse powers of the electron mass compared with the Maxwell term and are thus very small. Integrating out the electron field in the QED Lagrangian lead to a tower of new photon interactions which should be Lorentz and gauge invariant, and respect parity invariance. The first nontrivial photon interaction corresponds to dimension-eight operators, the Euler-Heisenberg Lagrangian, and reads

$$
\mathcal{L}_{\mathrm{EH}}=-\mathcal{F}+\frac{8}{45}\left(\frac{\alpha^{2}}{m_{e}^{4}}\right) \mathcal{F}^{2}+\frac{14}{45}\left(\frac{\alpha^{2}}{m_{e}^{4}}\right) \mathcal{G}^{2},
$$

with

$$
\begin{aligned}
\mathcal{F} & =\frac{1}{4} F_{\mu \nu} F^{\mu \nu}=\frac{1}{2}\left(\mathbf{B}^{2}-\mathbf{E}^{2}\right), \\
\mathcal{G} & =\frac{1}{8} \epsilon^{\mu \nu \lambda \rho} F_{\mu \nu} F^{\lambda \rho}=\mathbf{E} \cdot \mathbf{B},
\end{aligned}
$$


where $\mathbf{B}$ and $\mathbf{E}$ are the magnetic and electric fields, $\alpha=$ $e^{2} / 4 \pi$ the fine structure constant, $e$ the electron electric charge, and $\epsilon_{\mu \nu \lambda \rho}$ the totally antisymmetric tensor. The first term in Eq. (1), quadratic in the fields, is the classical Lagrangian corresponding to Maxwell's equations in vacuum. From it, one concludes that electromagnetic waves propagating in the vacuum cannot interact with each other, the superposition principle holds, and colliding light-bylight will not give rise to any scattering. However, this does not remain true once the corrections induced by the two last terms in Eq. (1) are included. At the loop level, electrodynamics is nonlinear even in vacuum. In that sense, observing e.g., the scattering of light by light would be a tremendous confirmation of the quantum nature of QED.

Another consequence of the nonlinearities in Eq. (1) is the so-called vacuum magnetic birefringence. Two photons interact with an external field and this leads, in vacuum, to magnetic birefringence, namely to different indices of refraction for light polarized parallel and perpendicular to an external magnetic field. This property of the vacuum has never been observed, despite many dedicated searches. For example, attempts were made to measure the change of the polarization of a laser beam passing through an external strong magnetic field [16-18]. The PVLAS [19] experiment is another approach to detect the vacuum birefringence, by measuring the degree of polarization of visible light from a magnetar, i.e., a neutron star whose magnetic field is presumably very large $\left(B \sim 10^{13} G\right)$. In that case, there is also an interesting interplay with well-motivated axionlike scenarios that could enhance the QED predictions (see e.g., [20]).

When discussing the Euler-Heisenberg result, one should also mention that the Born-Infeld (BI) electrodynamics [21] contains similar nonlinear corrections to the Maxwell theory, at least from a classical point of view. It was motivated by the idea that there should be an upper limit on the strength of the electromagnetic field. Nevertheless, BI electrodynamics is peculiar, since BI-type effective actions arise in many different contexts in superstring theory [22]. In heavy-ion collisions, the ATLAS data on light-by-light scattering can exclude the QED BI scale $\sim 100 \mathrm{GeV}$ [23]. It has been subsequently shown in Ref. [24] that the ATLAS data on $g g \rightarrow \gamma \gamma$ scattering enhances the sensitivity to $\lesssim 1 \mathrm{TeV}$ for the analogous dimension-8 operator scales (containing other combinations of gluon and electromagnetic fields). Searches for $\gamma \gamma$ production at possible future proton-proton colliders are an example of how one should complement the searches via dimension-6 SMEFT operators.

Returning to the purpose of this paper, generalizing $\mathrm{EH}$ to non-Abelian gauge bosons present several challenges. As a first step, all the effective interactions up to dimensioneight can be constructed solely relying on gauge invariance. The nonlinear nature of the field strength permits us to construct many more operators than for QED. Operators involving three field strengths arise already at the dimension-six level, and were constructed some time ago in Ref. [12]. The most general basis of operators for QCD, up to dimension eight and without imposing the gluon equation of motion (EOM), was described in Ref. [25].

Remains the task of actually computing the coupling constants of these operators, as induced by loops of heavy particles. To our knowledge, this has never been done before. To tackle this problem, there are two different approaches. First, the heavy particle field can be genuinely integrated out of the path integral. Several techniques are available to perform this integration and obtain the effective action at the one-loop level [26-32]. Though most powerful, the calculation has only been pushed up to the coefficients of dimension-six operators [28,29]. Another approach, which we will adopt in the present paper, is to actually compute the loop amplitudes, expand them in inverse power of the heavy particle mass, and match the result with that computed using effective interactions. Though most straightforward, several issues have to be addressed. Since the EOM should not be imposed to reproduce the generic effective action, loop amplitudes have to be computed off shell. But then, gauge invariance is not automatic since the amplitudes are not physical, and special care is needed to ensure a proper matching onto gauge invariant operators.

To illustrate our procedure and explain in details how to deal with these aspects, we start in the next section by rebuilding the well-known effective interactions of photons, as induced by loops of massive fermions, scalars, or vector bosons. In particular, we point out that using a nonlinear gauge is compulsory for the matching to succeed for massive vector fields, in agreement with Ref. [33]. Then, we generalize this computation to gluonic effective interactions in Sec. III, as induced by loops of massive fermions, scalars, or vector bosons in the fundamental representation of QCD. For the latter case, we use as prototypes the leptoquarks of the $S U(5)$ grand unified theory (GUT), quantized using a nonlinear gauge condition. As this is not fully standard, that construction is detailed in Appendix A.

Once the QCD case with heavy fields in the fundamental representation is fully under control, it is a simple matter to first generalize to arbitrary representations, and then to generic gauge groups. This is done in Sec. IV, where we discuss first the $S U(N)$ case, then show how to recover the previous results for $U(1)$ and $S U(3)$, and finally derive the mixed operators and their coefficients for a nonsimple gauge group like $U(1) \otimes S U(N)$ or $S U(N) \otimes S U(M)$. The most striking result of that section is that some operator combinations are never induced at one-loop, no matter the spin or representation of the heavy particle. For QCD, this means four instead of six operators are required to describe the four-gluon interaction, while only two instead of four operators are needed for the two gluon-two photon 
interaction. Throughout this section, the only technical difficulty is related to quartic Casimir invariants, which arise in the reduction of traces of four generators. From a group theory perspective, these invariants have been described in details before [34,35], but a more user-oriented review seems to be lacking. Therefore, we collect in Appendix B all the relevant information, as well as the explicit values of the quartic invariant for simple Lie algebras of interest for particle physics.

\section{PHOTON EFFECTIVE INTERACTIONS}

In the path integral formalism, the effective action is obtained by integrating out some heavy fields [36]. In general, this generates an infinite number of effective couplings among the remaining light fields. Renormalizability ensures that the effective couplings of dimension less than four can be absorbed into the light-field Lagrangian free parameters, while the other couplings are all finite and can be organized as a series in powers of the inverse of the heavy mass [37].

To set the stage, consider the QED generating functional

$$
\begin{aligned}
Z_{\mathrm{QED}}\left[J^{\mu}, \eta, \bar{\eta}\right]= & \int D A^{\mu} D \psi D \bar{\psi} \exp i \\
& \times \int d x\left(\mathcal{L}_{\mathrm{QED}}+\bar{\eta} \psi+\bar{\psi} \eta+J^{\mu} A_{\mu}\right),
\end{aligned}
$$

with

$$
\mathcal{L}_{\mathrm{QED}}=-\frac{1}{4} F_{\mu \nu} F^{\mu \nu}+\bar{\psi}(i \not D-m) \psi,
$$

and $D^{\mu}$ the usual covariant derivative. We omit the gauge fixing term and its associated ghosts. At very low energy, below $m$, only the photons are active. To construct the effective theory valid in that limit, the fermion field is integrated out. This can easily be carried out since the fermionic path integral is gaussian when the sources $\eta, \bar{\eta}$ are set to zero:

$$
\begin{aligned}
Z_{\mathrm{QED}}\left[J^{\mu}, 0,0\right]= & \int D A^{\mu} \exp i \int d x\left\{-\frac{1}{4} F_{\mu \nu} F^{\mu \nu}+J^{\mu} A_{\mu}\right\} \\
& \times \operatorname{det}(i \not D-m) \\
\equiv & \int D A^{\mu} \exp i \int d x\left(\mathcal{L}_{\text {eff }}+J^{\mu} A_{\mu}\right) .
\end{aligned}
$$

Exponentiating the determinant, the QED effective Lagrangian is then

$$
\mathcal{L}_{\text {eff }}=-\frac{1}{4} F_{\mu \nu} F^{\mu \nu}-i \operatorname{Tr} \ln (i \not D-m) .
$$

At this stage, several techniques are available to actually compute $\operatorname{det}(\not \not D-m)$ perturbatively, as an inverse mass expansion.
Probably the most universal and powerful way is using functional methods. Covariant calculation of the oneloop effective action can be obtained by using a heat kernel [38-40] to evaluate the effective action. This method utilizes a position space representation and is significantly more involved than the approach of Gaillard [26] and Cheyette [27] who introduced a manifestly gauge-covariant method of performing the calculation, using a covariant derivative expansion (CDE). This elegant method simplifies evaluating the quadratic term of the heavy fields in the path integral to obtain the low-energy EFT, and was revived recently in Ref. [28]. In particular, this work pointed out that under the assumption of degenerate particle masses one could evaluate the momentum dependence of the coefficients that factored out of the trace over the operator matrix structure, without specifying the specific UV model. In Ref. [29], it has been shown that this universality property can be extended without any assumptions on the mass spectrum, to obtain a universal result for the one-loop effective action for up to dimension-six operators. There the loop integrals have been computed for a general mass spectrum once and for all. This universal one-loop effective action $[29-32,41]$ is a general expression that may then be applied in any context where a one-loop path integral needs to be computed, as for example in matching new physics models to the Standard Model (SM) EFT. We should also mention the usefulness of the string-inspired technique for deriving effective actions [42-44].

However, in the present work, we wish to stick to the more pedestrian diagrammatic approach with external gauge fields, in which case one expands $\operatorname{det}(i \not D-m)$ as

$$
\mathcal{L}_{\text {eff }}=-\frac{1}{4} F_{\mu \nu} F^{\mu \nu}+i \sum_{n=1}^{\infty} \frac{e^{n}}{n} \operatorname{Tr}\left(\frac{1}{i \not \partial-m} \not A\right)^{n} .
$$

Graphically, this series is represented by the tower of oneloop 1PI diagrams shown in Fig. 1. The main advantage of expressing the effective action in terms of 1PI diagrams is that well-tested automatic tools are available to actually compute these loop amplitudes. In the present work, we will rely on the Mathematica packages FEYNARTS [45], FeynCalc [46], and Package X [47] (as implemented through FEYNHELPERS [48]).

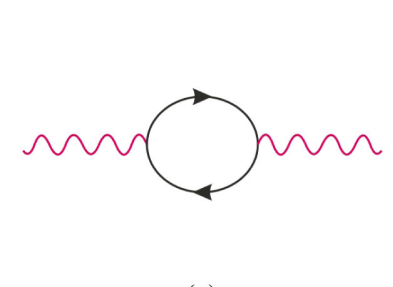

(a)

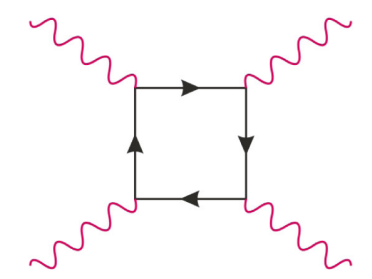

(b)
FIG. 1. Fermionic one-loop 1PI amplitudes generating the QED effective action up to dimension-eight operators. The six permutations of the photons are understood for diagram $(b)$. 
For QED, all the diagrams with an odd number of photons vanish because they are odd under charge conjugation (Furry's theorem [49]). Let us construct the effective couplings up to order $\mathrm{m}^{-4}$. First, the inverse-mass expansion of a charge-one fermion (of mass $m$ and quadrimomentum $p^{\mu}$ ) contribution to the photon vacuum polarization is

$$
\begin{aligned}
\Pi^{\mu \nu}\left(p^{2}\right)= & i \frac{8 e^{2}}{(4 \pi)^{2}}\left(g^{\mu \nu} p^{2}-p^{\mu} p^{\nu}\right) \\
& \times\left\{\frac{1}{6} D_{\varepsilon}+\frac{p^{2}}{30 m^{2}}+\frac{p^{4}}{280 m^{4}}+\mathcal{O}\left(p^{6} / m^{6}\right)\right\},
\end{aligned}
$$

with $D_{\varepsilon}=2 / \varepsilon-\gamma+\log 4 \pi \mu^{2} / m^{2}$. The corresponding effective interactions with two photons are

$$
\begin{aligned}
\mathfrak{Q}_{\mathrm{eff}}^{(0+2)}= & -\frac{1}{4}\left\{1+\frac{\alpha}{3 \pi} D_{\varepsilon}\right\} F_{\mu \nu} F^{\mu \nu}+\frac{\alpha}{60 \pi m^{2}} F_{\mu \nu} \square F^{\mu \nu} \\
& -\frac{\alpha}{560 \pi m^{4}} F_{\mu \nu} \square^{2} F^{\mu \nu}+\mathcal{O}\left(m^{-6}\right) .
\end{aligned}
$$

With four photons, the amplitude matches onto the two couplings

$\mathbf{\Omega}_{\mathrm{eff}}^{(4)}=\frac{\alpha^{2}}{90 m^{4}}\left(F_{\mu \nu} F^{\mu \nu}\right)^{2}+\frac{7 \alpha^{2}}{360 m^{4}}\left(F_{\mu \nu} \tilde{F}^{\mu \nu}\right)^{2}+\mathcal{O}\left(m^{-6}\right)$,

where the dual field strength is defined as $\tilde{F}^{\mu \nu}=\frac{1}{2} \varepsilon^{\mu \nu \rho \sigma} F_{\rho \sigma}$, so that $\left(F_{\mu \nu} \tilde{F}^{\mu \nu}\right)^{2}=2\left(F_{\mu \nu} F^{\mu \nu}\right)^{2}-4 F_{\mu \nu} F^{\nu \rho} F_{\rho \sigma} F^{\sigma \mu}$. The divergent term is the usual photon wave function renormalization, the first derivative term yields the Uehling interaction [50], and the Euler-Heisenberg effective couplings [1] are the nonderivative $\mathcal{O}\left(\mathrm{m}^{-4}\right)$ terms.

A word is in order concerning the derivative coupling. In most operator bases [12], it is eliminated using the equation of motion (EOM) as

$$
\begin{aligned}
F_{\mu \nu} \square F^{\mu \nu} & =F_{\mu \nu} \partial^{\rho} \partial_{\rho} F^{\mu \nu}=F_{\mu \nu} \partial^{\rho} \partial^{\mu} F_{\rho}{ }^{\nu}+F_{\mu \nu} \partial^{\rho} \partial^{\nu} F^{\mu}{ }_{\rho} \\
& =-2 \partial^{\mu} F_{\mu \rho} \partial_{\nu} F^{\nu \rho}=-2 j_{\nu} j^{\nu},
\end{aligned}
$$

where the Jacobi identity $\partial_{\mu} F_{\rho \nu}-\partial_{\rho} F_{\mu \nu}+\partial_{\nu} F_{\mu \rho}=0$ has been used in the first equality, followed by integration by part, and finally the equation of motion $\partial_{\mu} F^{\mu \nu}=j^{\nu}$. This makes sense physically, since the only impact of the Uehling potential is on the interaction between currents, at nonzero momentum transfer. Yet, in the QED effective theory considered here, all the fermions have been integrated out and $\partial_{\mu} F^{\mu \nu}=0$. This illustrate a generic feature of the effective action formalism, where all of the effects of the heavy fields are encoded into effective couplings among light fields at the path integral (i.e., quantum) level. At no stage are the light fields assumed on shell. So, some
TABLE I. Wilson coefficients of the effective photon operators for a scalar, fermion, and vector boson of electric charge $Q$. For the latter, the matching of 1PI amplitudes onto the $U(1)$ gauge-invariant operators of Eq. (13) is possible only when using a nonlinear gauge for the massive vector bosons, and the quoted values for $\alpha_{1,2,3}$ are specific to that gauge $[\kappa=1$ in Eqs. (16) and (17)].

\begin{tabular}{lccccc}
\hline \hline & $\alpha_{0}$ & $\alpha_{2}$ & $\alpha_{4}$ & $\gamma_{4,1}$ & $\gamma_{4,2}$ \\
\hline Scalar & $\frac{1}{2} D_{\varepsilon} Q^{2}$ & $-\frac{1}{8} Q^{2}$ & $\frac{3}{56} Q^{2}$ & $\frac{7}{32} Q^{4}$ & $\frac{1}{32} Q^{4}$ \\
Fermion & $2 D_{\varepsilon} Q^{2}$ & $-Q^{2}$ & $\frac{9}{14} Q^{2}$ & $\frac{1}{2} Q^{4}$ & $\frac{7}{8} Q^{4}$ \\
Vector & $-\frac{21 D_{\varepsilon}+2}{2} Q^{2}$ & $\frac{37}{8} Q^{2}$ & $-\frac{159}{56} Q^{2}$ & $\frac{261}{32} Q^{4}$ & $\frac{243}{32} Q^{4}$ \\
\hline \hline
\end{tabular}

effective interactions may actually never contribute to physical processes, even though they are required to fully encode the underlying dynamics of the heavy field.

At this stage, it should also be clear that the effective couplings can be constructed a priori. Using only the requirement of QED gauge invariance, the most general basis is

$$
\begin{aligned}
\mathfrak{Q}_{\mathrm{eff}}= & -\frac{1}{4}\left\{1+\alpha_{0} \frac{e^{2}}{4 ! \pi^{2}}\right\} F_{\mu \nu} F^{\mu \nu}+\alpha_{2} \frac{e^{2}}{5 ! \pi^{2} m^{2}} \partial^{\mu} F_{\mu \nu} \partial_{\rho} F^{\rho \nu} \\
& +\alpha_{4} \frac{e^{2}}{6 ! \pi^{2} m^{4}} \partial^{\mu} F_{\mu \nu} \square \partial_{\rho} F^{\rho \nu}+\gamma_{4,1} \frac{e^{4}}{6 ! \pi^{2} m^{4}}\left(F_{\mu \nu} F^{\mu \nu}\right)^{2} \\
& +\gamma_{4,2} \frac{e^{4}}{6 ! \pi^{2} m^{4}}\left(F_{\mu \nu} \tilde{F}^{\mu \nu}\right)^{2}+\mathcal{O}\left(m^{-6}\right) .
\end{aligned}
$$

The derivative operators are rewritten in a form that makes the EOM manifest. This will prove useful when comparing with the non-Abelian results in the next section, for which this choice of operator basis is far more convenient. The nomenclature adopted throughout the paper is to denote by $\alpha_{i}, \beta_{i}, \gamma_{i}$ the two, three, four-field strength couplings of inverse mass dimension $i$. Only the specific values of these coefficients encode the information about the heavy field, and we give in Table I the results for a scalar, fermion, and vector boson. Note that the sole purpose of the rather unconventional normalization of the coefficients in Eq. (13) is to increase the readability of Table I. It is designed to make the coefficients appear as simple $\mathcal{O}(1)$ fractions for the fermion case.

The calculation in the scalar case is very similar to that for fermions and present no particular difficulty (see Fig. 2). On the other hand, that for vectors circulating in the loop is far less straightforward. Let us take the SM, where the electroweak gauge bosons acquire their masses through the Higgs mechanism. Working in the 't HooftFeynman gauge, the amplitude does not satisfy the QED ward identities when the photons are off shell. Consequently, the four-photon amplitude matches onto the local $\mathcal{O}\left(\mathrm{m}^{-4}\right)$ effective operators only when the four photons are on shell [51], and the usual procedure to 


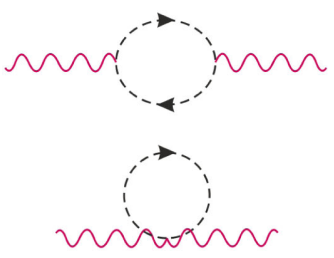

(a)
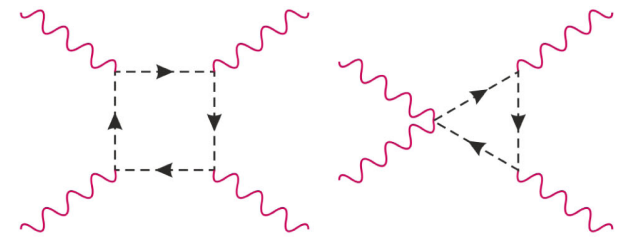

(b)

FIG. 2. Scalar one-loop 1PI amplitudes generating the QED effective action up to dimension-eight operators. Permutations of the photons are understood for diagrams (b). For massive vector bosons, the topologies are the same but one should also include the appropriate would-be Goldstone and ghost diagrams.

construct the effective action breaks down. The problem originates in the gauge-fixing procedure. In the usual $R_{\xi}$ gauge, one adds the term

$$
\mathfrak{\Omega}_{\text {gauge-fixing }}^{R_{\xi}, \text { linear }}=-\frac{1}{\xi}\left|\partial^{\mu} W_{\mu}^{+}+\xi M_{W} \phi^{+}\right|^{2},
$$

with $\phi^{ \pm}$the would-be Goldstone (WBG) scalars associated to $W^{ \pm}$, and this explicitly breaks $U(1)_{\mathrm{QED}}$. Though the photon vacuum polarization remains transverse and matches onto the effective operators in Eq. (13), the off shell four-photon amplitude is not gauge invariant and requires more operators already at the $\mathcal{O}\left(\mathrm{m}^{-2}\right)$ [52]. Of course, physical processes have to be gauge invariant, so this should have no consequence. But in practice, adding nongauge invariant operators in the effective Lagrangian is not very appealing. One could attempt to solve this problem by working in the unitary gauge, for which the $W$ couplings to the photon derive from

$$
\begin{aligned}
\mathcal{L}_{U-\text { gauge }}= & -\frac{1}{2}\left(D_{\mu} W_{\nu}^{+}-D_{\nu} W_{\nu}^{+}\right)\left(D^{\mu} W^{-\nu}-D^{\nu} W^{-\mu}\right) \\
& +i e F^{\mu \nu} W_{\mu}^{+} W_{\nu}^{-}+M_{W}^{2} W_{\mu}^{+} W^{-\mu},
\end{aligned}
$$

where $D_{\mu} W_{\nu}^{ \pm}=\partial_{\mu} W_{\nu}^{ \pm} \mp i e A_{\mu} W_{\nu}^{ \pm}$. The magnetic moment term $F^{\mu \nu} W_{\mu}^{+} W_{\nu}^{-}$, gauge-invariant by itself, is fixed by the underlying $S U(2)_{L}$ gauge symmetry. As shown in Ref. [53], its presence ensures a proper high-energy behavior for scattering amplitudes. However, this is not sufficient to ensure a correct behavior off shell, and the matching fails again [54].

A better way to proceed is to enforce a nonlinear gauge condition where $\partial^{\mu} W_{\mu}^{ \pm} \rightarrow D^{\mu} W_{\mu}^{ \pm}=\partial^{\mu} W_{\mu}^{ \pm} \pm i e A^{\mu} W_{\mu}^{ \pm}$in Eq. (14). This closely parallels the constraint one needs to impose to construct the CDE [28]. In the diagrammatic approach, as shown in Ref. [33], the four-photon amplitude is then gauge invariant, even off shell. We checked this explicitly using the dedicated FEYNARTS model file [55] for the SM in the nonlinear gauge, and indeed found a consistent off shell matching on the Euler-Heisenberg operators. The result in that gauge for all the coefficients is shown in Table I. It should be clear though that the first three coefficients are gauge-dependent, and only $\gamma_{4,1}$ and $\gamma_{4,2}$ are physical. To investigate this feature, let us set the gauge fixing term as $[56,57]$

$\mathfrak{Q}_{\text {gauge-fixing }}^{\text {nonlinear }}=-\frac{1}{\xi}\left|\partial^{\mu} W_{\mu}^{+}+i \kappa e A^{\mu} W_{\mu}^{+}+\xi M_{W} \phi^{+}\right|^{2}$,

which permits us to interpolate between the linear $(\kappa=0)$ and the $U(1)$-gauge-invariant nonlinear $(\kappa=1)$ gauge. The inverse-mass expansion of the photon vacuum polarization in the 't Hooft-Feynman gauge $(\xi=1)$ then gives $\kappa$ dependent coefficients:

$$
\begin{aligned}
& \alpha_{0}=-\frac{12 \kappa+9}{2} D_{\varepsilon}-1, \quad \alpha_{2}=\frac{20 \kappa+17}{8}, \\
& \alpha_{4}=-\frac{84 \kappa+75}{56}
\end{aligned}
$$

Of course, these gauge dependences are unphysical. At very low energy, when the photon remains as the only active degree of freedom, the first coefficient is absorbed into the photon field as the wave function renormalization constant while the other two do not contribute since $\partial_{\mu} F^{\mu \nu}=0$. If some fields remain active such that $\partial_{\mu} F^{\mu \nu}=j^{\nu} \neq 0$, then other types of processes are also present. In that case, the $\alpha_{2}$ operator should be eliminated in favor of the dimension-six $j_{\mu} j^{\mu} / \mathrm{m}^{2}$ operator, for which other diagrams occur. In the $\mathrm{SM}$, even if the fields in the current $j^{\mu}$ are not coupled directly to the $W^{ \pm}$, they are necessarily coupled to the $Z$ boson. The $\kappa$ dependence of the $W^{ \pm}$contributions to the $Z \gamma$ and $Z Z$ vacuum polarization [58] must cancel that of $\alpha_{2}$, so that the coefficient of the $j_{\mu} j^{\mu} / \mathrm{m}^{2}$ operator ends up gaugeinvariant and physical. The conclusion is thus that in the SM, it is not consistent to define the Uehling potential in terms of the $F_{\mu \nu} \square F^{\mu \nu}$ operator, and one must use the effective fourfermion operators instead. After all, this is rather natural since the Uehling potential is only relevant when some fermion fields remain active.

\section{GLUON EFFECTIVE INTERACTIONS}

The effective action for gluon fields is constructed in the same way as for photons, using the diagrammatic approach. For example, integrating out a heavy fermion generates 


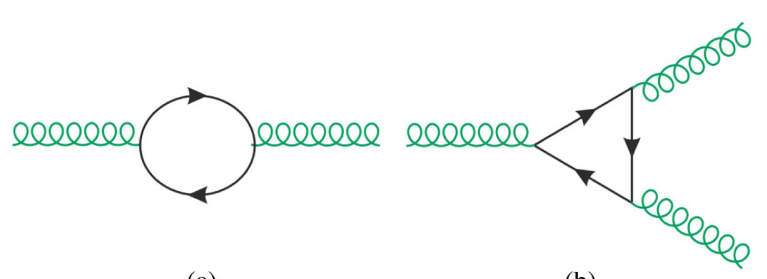

(a) (b)

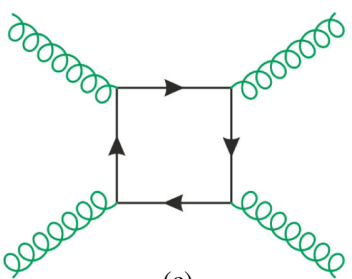

(c)

FIG. 3. Fermionic one-loop 1PI amplitudes generating the gluonic effective action. Permutations of the gluons are understood for diagrams (b) and (c). As for QED in Fig. 2, additional diagrams are understood for the scalar and vector case.

$$
\begin{aligned}
\mathcal{L}_{\text {eff }} & =-\frac{1}{4} G_{\mu \nu}^{a} G^{a, \mu \nu}-i \operatorname{Tr} \ln (i \not D-m) \\
& =-\frac{1}{4} G_{\mu \nu}^{a} G^{a, \mu \nu}+i \sum_{n=1}^{\infty} \frac{e^{n}}{n} \operatorname{Tr}\left(\frac{1}{i \not \supset-m} G^{a} T^{a}\right)^{n},
\end{aligned}
$$

where $T^{a}$ are the $S U(3)$ generators, and the trace carries over both Dirac and color space. This generates the series of 1PI diagrams shown in Fig. 3 where, contrary to QED, the odd-number of gluon amplitudes do not vanish. Another difference with respect to QED is the nonlinear nature of the field strength, which blurs the relationship between the leading inverse-mass power of a given diagram and the number of external gluons. The most striking consequence is that the three and four-gluon diagrams are not finite. Actually, since these infinities both correspond to the renormalization of the same operator $G_{\mu \nu}^{a} G^{a, \mu \nu}$, they must be coherent with that obtained from the two-gluon vacuum polarization. Let us see how this happens in more details.

As a first step in the calculation of the effective action, let us construct the most general basis of operators up to $\mathcal{O}\left(m^{-4}\right)$. With two field strengths, the operators are simple generalizations of those for QED:

$$
\begin{aligned}
\mathfrak{R}_{\mathrm{eff}}^{(0+2)}= & -\frac{1}{4}\left\{1+\alpha_{0} \frac{g_{S}^{2}}{4 ! \pi^{2}}\right\} G_{\mu \nu}^{a} G^{a, \mu \nu} \\
& +\alpha_{2} \frac{g_{S}^{2}}{5 ! \pi^{2} m^{2}} D^{\nu} G_{\nu \mu}^{a} D_{\rho} G^{a, \rho \mu} \\
& +\alpha_{4} \frac{g_{S}^{2}}{6 ! \pi^{2} m^{4}} D^{\nu} G_{\nu \mu}^{a} D^{2} D_{\rho} G^{a, \rho \mu}
\end{aligned}
$$

where $G_{\mu \nu}^{a}=\partial_{\mu} A_{\nu}^{a}-\partial_{\nu} A_{\mu}^{a}+g f^{a b c} A_{\mu}^{b} A_{\nu}^{c}$ and $D_{\rho} G_{\mu \nu}^{a}=$ $\left(\partial_{\rho} \delta^{a c}+g f^{a b c} G_{\rho}^{b}\right) G_{\mu \nu}^{c}$. To see that there can be only one derivative operator per inverse-mass order [12], first remark that all the derivatives can be move to act on one of the field strength by partial integration. Then, only one ordering of the covariant derivatives is relevant since commuting them generates an additional field strength, $\left[D^{\rho}, D^{\sigma}\right] G_{\mu \nu}^{a}=$ $g f^{a b c} G_{\rho \sigma}^{b} G_{\mu \nu}^{c}$. Finally, combining this with the Bianchi identity

$$
D_{[\mu} G_{\rho \sigma]}^{a}=D_{\mu} G_{\rho \sigma}^{a}+D_{\rho} G_{\sigma \mu}^{a}+D_{\sigma} G_{\mu \rho}^{a}=0
$$

these operators can be written as manifestly vanishing under the EOM for the field strength, $D^{\mu} G_{\mu \nu}^{a}=0$. Let us stress though that the EOM are not used at any stage, since using them would render the matching impossible.

With three-gluon field strengths, there is only one operator at $\mathcal{O}\left(\mathrm{m}^{-2}\right)$ but many at $\mathcal{O}\left(\mathrm{m}^{-4}\right)$. However, upon partial integration, use of the Bianchi identity, and discarding terms involving four or more field strengths, only two inequivalent contractions remain [59]. Here again, we choose them to be manifestly vanishing under the field strength EOM:

$$
\begin{aligned}
\mathfrak{Q}_{\mathrm{eff}}^{(3)}= & \beta_{2} \frac{g_{S}^{3}}{5 ! \pi^{2} m^{2}} f^{a b c} G_{\mu}^{a \nu} G_{\nu}^{b \rho} G_{\rho}^{c \mu} \\
& +\beta_{4,1} \frac{g_{S}^{3}}{6 ! \pi^{2} m^{4}} f^{a b c} G^{a, \mu \nu} D^{\alpha} G_{\mu \nu}^{b} D^{\beta} G_{\alpha \beta}^{c} \\
& +\beta_{4,2} \frac{g_{S}^{3}}{6 ! \pi^{2} m^{4}} f^{a b c} G^{a, \mu \nu} D^{\alpha} G_{\alpha \mu}^{b} D^{\beta} G_{\beta \nu}^{c} .
\end{aligned}
$$

At the four-field strength level, the operators up to $\mathcal{O}\left(\mathrm{m}^{-4}\right)$ contain no covariant derivatives. To reach a minimal number of operators, we use the generalization of the QED identity:

$$
\begin{aligned}
G_{\mu \nu}^{a} \tilde{G}^{b, \mu \nu} G_{\rho \sigma}^{c} \tilde{G}^{d, \rho \sigma}= & G_{\mu \nu}^{a} G^{c, \mu \nu} G_{\rho \sigma}^{b} G^{d, \rho \sigma}+G_{\mu \nu}^{a} G^{d, \mu \nu} G_{\rho \sigma}^{b} G^{c, \rho \sigma} \\
& -4 G_{\mu \nu}^{a} G^{c, \nu \rho} G_{\rho \sigma}^{b} G^{d, \sigma \mu}
\end{aligned}
$$

and note that no contractions with the totally symmetric tensor $d^{a b c}$ occurs because those are reduced using (see Appendix B)

$3 d^{a b e} d^{c d e}=\delta^{a c} \delta^{b d}-\delta^{a b} \delta^{c d}+\delta^{a d} \delta^{b c}+f^{a c e} f^{b d e}+f^{a d e} f^{b c e}$.

Contractions with both $f$ and $d$ tensors vanish identically owing to their mixed symmetry properties. This leaves six $\mathcal{O}\left(m^{-4}\right)$ operators for $\mathfrak{Q}_{\text {eff }}^{(4)}$ : 


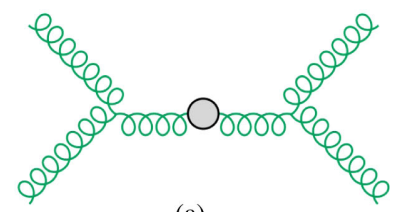

(a)

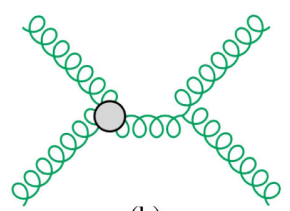

(b)

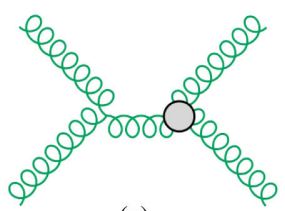

(c)

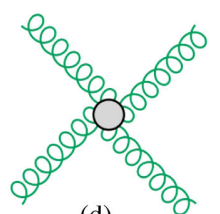

(d)

FIG. 4. The four basic $s$-channel topologies for the gluon-gluon scattering amplitude. That for the $t$ - and $u$-channel are understood. The grey disks represent the insertion of the effective action vertices.

$$
\begin{aligned}
\mathfrak{Q}_{\mathrm{eff}}^{(4)}= & \gamma_{4,1} \frac{g_{S}^{4}}{6 ! \pi^{2} m^{4}} G_{\mu \nu}^{a} G^{a, \mu \nu} G_{\rho \sigma}^{b} G^{b, \rho \sigma} \\
& +\gamma_{4,2} \frac{g_{S}^{4}}{6 ! \pi^{2} m^{4}} G_{\mu \nu}^{a} \tilde{G}^{a, \mu \nu} G_{\rho \sigma}^{b} \tilde{G}^{b, \rho \sigma} \\
& +\gamma_{4,3} \frac{g_{S}^{4}}{6 ! \pi^{2} m^{4}} G_{\mu \nu}^{a} G^{b, \mu \nu} G_{\rho \sigma}^{a} G^{b, \rho \sigma} \\
& +\gamma_{4,4} \frac{g_{S}^{4}}{6 ! \pi^{2} m^{4}} G_{\mu \nu}^{a} \tilde{G}^{b, \mu \nu} G_{\rho \sigma}^{a} \tilde{G}^{b, \rho \sigma} \\
& +\gamma_{4,5} \frac{g_{S}^{4}}{6 ! \pi^{2} m^{4}} f^{a b e} f^{c d e} G_{\mu \nu}^{a} G^{c, \mu \nu} G_{\rho \sigma}^{b} G^{d, \rho \sigma} \\
& +\gamma_{4,6} \frac{g_{S}^{4}}{6 ! \pi^{2} m^{4}} f^{a b e} f^{c d e} G_{\mu \nu}^{a} \tilde{G}^{c, \mu \nu} G_{\rho \sigma}^{b} \tilde{G}^{d, \rho \sigma} .
\end{aligned}
$$

This basis corresponds to that in Ref. [25], but for a slightly different numbering and replacement of dual tensors via Eq. (23).

The non-Abelian nature of QCD makes the effective action expansion quite different from the QED case. The operators vanishing under the EOM have to be kept because they contribute to several off shell 1PI diagrams. For example, the $D^{\nu} G_{\nu \mu}^{a} D_{\rho} G^{a, \rho \mu}$ operator occurs in the two, three, and four-gluon off shell 1PI diagrams of Fig. 3 simply because of the non-Abelian terms present in the gluon field strengths. On the other hand, for a physical process involving external on shell gluons, these operators should not contribute, and the basis could be simplified. Let us check this in the simplest case, which is the gluon-gluon scattering amplitude

$$
\begin{aligned}
\mathcal{A}\left(g\left(p_{1}, \varepsilon_{p_{1}}^{\mu_{1}}\right) g\left(p_{2}, \varepsilon_{p_{2}}^{\mu_{2}}\right)\right. & \left.\rightarrow g\left(p_{3}, \varepsilon_{p_{3}}^{\mu_{3}}\right) g\left(p_{4}, \varepsilon_{p_{4}}^{\mu_{4}}\right)\right) \\
& =\varepsilon_{p_{1}}^{\mu_{1}} \varepsilon_{p_{2}}^{\mu_{2}} \varepsilon_{p_{3}}^{\mu_{3} *} \varepsilon_{p_{4}}^{\mu_{4} *} \mathcal{M}_{\mu_{1} \mu_{2} \mu_{3} \mu_{4}} .
\end{aligned}
$$

Computing this amplitude using the effective Lagrangian up to $\mathcal{O}\left(\mathrm{m}^{-4}\right)$, the basic topologies to consider are shown in Fig. 4. Besides the four point local terms, we must add the nonlocal contributions from the three-gluon and two-gluon operators, as well as the wave function corrected tree-level term. We observe:

(i) The wave function correction is automatically accounted for through a rescaling of the field and coupling constant $g_{S}$.

(ii) The $\boldsymbol{R}_{\text {eff }}^{(2)}$ operators contribute to all topologies, $\boldsymbol{\Omega}_{\text {eff }}^{(3)}$ operators to $(b-d)$ topologies, and $\boldsymbol{\Omega}_{\mathrm{eff}}^{(4)}$ to the $(d)$ topology only. (iii) For the EOM operators, these topologically distinct contributions precisely cancel each other. These operators thus play no role for physical processes.

(iv) Independently for each non-EOM operator $Q_{i}$, the sum of the contributions $\mathcal{M}_{\mu_{1} \mu_{2} \mu_{3} \mu_{4}}\left(Q_{i}\right)$ satisfy the four Ward identities $p_{k}^{\mu_{k}} \mathcal{M}_{\mu_{1} \mu_{2} \mu_{3} \mu_{4}}\left(Q_{i}\right)=0, k=1$, $2,3,4$.

The fact that EOM operators drop out of the full physical amplitude can be easily understood qualitatively. For example, taking the dimension-six $\mathfrak{R}_{\text {eff }}^{(2)}$ operator $D^{\nu} G_{\nu \mu}^{a} D_{\rho} G^{a, \rho \mu}$ and expanding the covariant derivatives, we get

$$
\begin{aligned}
D^{\nu} G_{\nu \mu}^{a} D_{\rho} G^{a, \rho \mu}= & \partial^{\nu} G_{\nu \mu}^{a} \partial_{\rho} G^{a, \rho \mu}+g f^{a b c} G^{b, \nu} G_{\nu \mu}^{c} \partial_{\rho} G^{a, \rho \mu} \\
& +g f^{a b c} \partial^{\nu} G_{\nu \mu}^{a} G_{\rho}^{b} G^{c, \rho \mu} \\
& +g^{2} f^{a b c} f^{a d e} G^{b, \nu} G_{\nu \mu}^{c} G_{\rho}^{d} G^{e, \rho \mu}
\end{aligned}
$$

Replacing the field strength as $G_{\mu \nu}^{a} \rightarrow \partial_{\mu} G_{\nu}^{a}-\partial_{\nu} G_{\mu}^{a}$, these four terms are precisely those entering the four topologies in Fig. 4. We can see that the cancellation occurs because the gluon propagator poles are precisely compensated by the momentum factors arising from the LO three-gluon vertex and from the derivatives in the first three terms of Eq. (27). A similar reasoning can be applied to the non-Abelian terms in the field strengths, which cancel out similarly.

Let us now compute explicitly the coefficients of the effective operators for a fermion, scalar, or vector in the fundamental representation. Generically, the procedure is as follows: Starting with the vacuum polarization graph [Fig. 3(a)], we fix the $\alpha_{0,2,4}$ coefficients. Then, the threepoint 1PI loop amplitudes [Fig. 3(b)] generate again the $\mathbf{R}_{\text {eff }}^{(2)}$ operators together with that of $\boldsymbol{\Omega}_{\text {eff }}^{(3)}$ and thus fix $\beta_{2}$, $\beta_{4,1}$, and $\beta_{4,2}$. As a side effect, the basis chosen for $\boldsymbol{Q}_{\mathrm{eff}}^{(0+2)}$ thus affects the three Wilson coefficients of $\boldsymbol{\Omega}_{\mathrm{eff}}^{(3)}$. Finally, the four-point 1PI amplitudes [Fig. 3(c)] match over the local four-gluon terms extracted from $\mathfrak{\Omega}_{\text {eff }}^{(0+2+3+4)}$, and given the coefficients obtained in the first two steps, fix the six $\gamma_{4, i}$ coefficients. The final results for the coefficients are given in Table II. They agree with Ref. [28] for dimension-six operators.

This procedure is rather straightforward for scalars and fermions circulating in the loop, and only marginally more complicated than in the QED case of Sec. II. We checked our computation using the SM and minimal 
TABLE II. Wilson coefficients of the effective gluonic operators for a scalar, fermion, and vector boson in the fundamental representation. This corresponds for example to the contributions of squarks in the MSSM, or heavy quarks in the SM. For the coefficients in the vector case, we use the leptoquark gauge fields of the minimal $S U(5)$ GUT model, quantized using a nonlinear gauge fixing procedure (see Appendix A).

\begin{tabular}{|c|c|c|c|c|c|c|}
\hline & $\alpha_{0}$ & $\alpha_{2}$ & $\alpha_{4}$ & $\beta_{2}$ & $\beta_{4,1}$ & $\beta_{4,2}$ \\
\hline Scalar & $\frac{1}{4} D_{\varepsilon}$ & $-\frac{1}{16}$ & $\frac{3}{112}$ & $\frac{1}{48}$ & $-\frac{1}{28}$ & 0 \\
\hline Fermion & $D_{\varepsilon}$ & $-\frac{1}{2}$ & $\frac{9}{28}$ & $-\frac{1}{24}$ & $\frac{1}{14}$ & $-\frac{3}{4}$ \\
\hline \multirow[t]{2}{*}{ Vector } & $-\frac{21 D_{\varepsilon}+2}{4}$ & $\frac{37}{16}$ & $-\frac{159}{112}$ & $\frac{1}{16}$ & $-\frac{3}{28}$ & 3 \\
\hline & $\gamma_{4,1}$ & $\gamma_{4,2}$ & $\gamma_{4,3}$ & $\gamma_{4,4}$ & $\gamma_{4,5}$ & $\gamma_{4,6}$ \\
\hline Scalar & $\frac{7}{768}$ & $\frac{1}{768}$ & $\frac{7}{384}$ & $\frac{1}{384}$ & $\frac{1}{96}$ & $\frac{1}{672}$ \\
\hline Fermion & $\frac{1}{48}$ & $\frac{7}{192}$ & $\frac{1}{24}$ & $\frac{7}{96}$ & $\frac{1}{96}$ & $\frac{19}{672}$ \\
\hline Vector & $\frac{87}{256}$ & $\frac{81}{256}$ & $\frac{87}{128}$ & $\frac{81}{128}$ & $-\frac{3}{32}$ & $-\frac{27}{224}$ \\
\hline
\end{tabular}

supersymmetric Standard Model (MSSM) FEYNARTS models, using quarks or squarks as representative particles in the fundamental representation. For vector particles, the calculation is far more challenging. First, we must construct a consistent model involving a massive vector field in the fundamental representation of QCD. Second, we know from the QED case that working in the unitary gauge does not work, and even introducing an appropriate Higgs mechanism to make these vectors massive is not sufficient. Some generalization of the nonlinear gauge has to be designed to preserve the QCD symmetry throughout the quantization, otherwise the 1PI off shell amplitudes cannot be matched onto gauge invariant operators. This is particularly annoying here since the three gluon 1PI amplitudes kinematically vanish on shell.

To proceed, our strategy is to use the minimal $S U(5)$ GUT model, spontaneously broken by an adjoint Higgs scalar down to the (unbroken) SM gauge group. Twelve of the $S U(5)$ gauge bosons become massive in the process, and those fields have precisely the quantum numbers we need. The weak doublet of leptoquarks $(X, Y)$ transforms as color antitriplets, so integrating them out generate the effective gluonic operators. Note that we do not need the second breaking stage down to $S U(3)_{C} \otimes U(1)_{e m}$. In Appendix A, we describe in some details the minimal $S U$ (5) GUT model, along with its quantization using nonlinear gauge fixing terms for the $X$ and $Y$ gauge bosons. Denoting by $H_{X}^{k}$ and $H_{Y}^{k}$ the WBG scalars associated to $X_{\mu}^{k}$ and $Y_{\mu}^{k}$, the main point is to modify the usual $R_{\xi}$ gauge fixing terms

$$
\begin{aligned}
\mathcal{L}_{\mathrm{gf}}= & -\frac{1}{\xi}\left|\partial^{\mu} X_{\mu}^{k+}-i \xi M_{X Y} H_{X}^{k+}\right|^{2}-\frac{1}{\xi} \mid \partial^{\mu} Y_{\mu}^{k+} \\
& -\left.i \xi M_{X Y} H_{Y}^{k+}\right|^{2}+\cdots
\end{aligned}
$$

by replacing the derivative by

$$
\begin{aligned}
\partial^{\mu} X_{\mu}^{i+} \rightarrow & \partial^{\mu} X_{\mu}^{i+}-i g_{5}\left(-\alpha_{G} X_{\nu}^{j+} T_{j i}^{a} G_{\mu}^{a}+\frac{\alpha_{W}}{2} W_{\mu}^{3} X_{\nu}^{i+}\right. \\
& \left.+\frac{\alpha_{W}}{\sqrt{2}} W_{\mu}^{+} Y_{\nu}^{i+}+\alpha_{B} \sqrt{\frac{5}{12}} B_{\mu} X_{\nu}^{i+}\right), \\
\partial^{\mu} Y_{\nu}^{i+} \rightarrow & \partial^{\mu} Y_{\mu}^{i \pm}-i g_{5}\left(-\alpha_{G} Y_{\nu}^{j+} T_{j i}^{a} G_{\mu}^{a}-\frac{\alpha_{W}}{2} W_{\mu}^{3} Y_{\nu}^{i+}\right. \\
& \left.+\frac{\alpha_{W}}{\sqrt{2}} W_{\mu}^{-} X_{\nu}^{i+}+\alpha_{B} \sqrt{\frac{5}{12}} B_{\mu} Y_{\nu}^{i+}\right),
\end{aligned}
$$

where $T^{\alpha}$ are the $S U(3)$ generators for the fundamental representation, and $i, j, k$ the corresponding indices. The gauge parameters $\alpha_{G}, \alpha_{W}, \alpha_{B}$ interpolate between the 't Hooft-Feynman gauge $\alpha_{G}=\alpha_{W}=\alpha_{B}=0$ and the nonlinear gauge $\alpha_{G}=\alpha_{W}=\alpha_{B}=1$, when the above terms coincide with $D^{\mu} X_{\mu}^{i+}$ and $D^{\mu} Y_{\mu}^{i+}$. In that limit, the SM gauge symmetries are preserved, exactly like the $U(1)_{e m}$ in the SM in the nonlinear gauge. Technically, it should be remarked also that this gauge has the nice feature of drastically reducing the number of diagrams for a given process [57]. Indeed, remember that the purpose of the usual $R_{\xi}$ gauge is to get rid of the mixing terms like $X_{\mu}^{k} \partial^{\mu} H_{X}^{k}$. But when the vector is charged under some remaining unbroken symmetries, this term is necessarily of the form $X_{\mu}^{k} D^{\mu} H_{X}^{k}$ since it arises from the Higgs scalar kinetic term which is invariant under the unbroken symmetries. With the nonlinear gauge, all these terms cancel out, leaving no $X-V_{\mathrm{SM}}-H_{X}$ couplings. As a result, all the mixed loops where the massive vector occurs alongside its WBG boson disappear, and given the large number of diagrams, this is very welcome.

To actually perform the computation, we again use FEYNARTS [45] but with a custom $S U(5)$ model file. The calculation then proceeds without particular difficulty, and gives the coefficients quoted in Table II. Several comments are in order:

(i) The matching works only for $\alpha_{G}=\alpha_{W}=\alpha_{B}=1$. Without this condition, nongauge-invariant operators are required. Note that out of a total of 207 irreducible four-gluon diagrams, the gauge conditions $\alpha_{G}=$ $\alpha_{W}=\alpha_{B}=1$ leaves only 21 gauge-boson loops, $21 \mathrm{WBG}$ loops, and 42 ghost loops. The disappearance of mixed loops therefore reduces the number of diagrams by more than a factor of two.

(ii) Many of the properties discovered in Ref. [33] for photons survive to the non-Abelian generalization: the ghost and WBG contributions are separately gauge invariant when $\alpha_{G}=\alpha_{W}=\alpha_{B}=1$. Actually, matching separately the $H_{X}^{k}$ contributions on the effective operators reproduce the coefficients for the scalar case in Table II, while matching the $c_{X}$ and $c_{X}^{\dagger}$ ghost contributions gives -2 times the scalar 
coefficients of Table II. With the nonlinear gauge, the ghosts behave exactly like scalar particles, but for the fermi statistics.

(iii) As a check, we computed the full physical gluongluon scattering amplitude keeping the gauge parameter $\alpha_{G}$ arbitrary. On shell and when both 1PI and non-1PI topologies are included, the only remaining $\alpha_{G}$ dependence can be absorbed into a wave function correction. In other words, the inverse-mass expansion of the full amplitude matches onto the non-EOM operators, and except for $\alpha_{0}$, their coefficients are gauge-independent and physical, as they should.

(iv) To further check our results, we computed the 1PI diagrams with two, three, and four external $S U(2)_{L}$ bosons. Since $S U(2)_{L}$ is kept unbroken, and since $(X, Y)$ form an $S U(2)_{L}$ doublet, we can use the same operator basis as for gluons, up to obvious substitutions, and found again the coefficients in Table II.

(v) Finally, we also computed the effective operators involving two and four $U(1)_{Y}$ gauge bosons, and recover the same results as in Table I for the $W^{ \pm}$ contribution in the nonlinear gauge to photon effective operators.

To close this section, the same cautionary remark as for the Uehling operator should be repeated here for EOM gluonic operators. Those play no role for on shell gluon processes, but do contribute when other fields like light quarks remain active. However, in that case, it is compulsory to include also all the effective operators involving quark fields. Though the EOM operators are gauge invariant by construction, their coefficients are not gauge invariant by themselves. For instance, the gauge chosen for the $X_{\mu}^{k}$ and $Y_{\mu}^{k}$ fields does affect their values [Eq. (17) remains valid for the gluonic vacuum polarization]. In a phenomenological study, it would thus make no sense to consider for example the $D^{\nu} G_{\nu \mu}^{a} D_{\rho} G^{a, \rho \mu}$ operator without including all the fourquark operators. Taking again $S U(5)$, it is clear that $X_{\mu}^{k}$ and $Y_{\mu}^{k}$ loops would contribute to both $D^{\nu} G_{\nu \mu}^{a} D_{\rho} G^{a, \rho \mu}$ and fourquark operators, and only their combination would result in a gauge-invariant physical result at the dimension-six level. As an aside, it should be mentioned also that the gaugedependent coefficient of the $D^{\nu} G_{\nu \mu}^{a} D_{\rho} G^{a, \rho \mu}$ operator quoted in Table II agrees with that in Ref. [28]; the CDE computation being done in the same nonlinear gauge.

\section{IV. $S U(N)$ EFFECTIVE INTERACTIONS}

The computation done in the case of QCD can be extended to arbitrary representations of other Lie groups. For that, it suffices to replace the traces over the fundamental generators of $S U(3)$ occurring for each of the 1PI diagrams of the previous section by traces over generators in some generic representation $\mathbf{R}$. Our notations along with various group-theoretic results are collected in Appendix B. In this section, for definiteness, we refer to $S U(N)$ gauge group, but the results are trivially extended to other Lie algebras.

Specifically, the vacuum polarization is tuned by $\operatorname{Tr}\left(T_{\mathbf{R}}^{a} T_{\mathbf{R}}^{b}\right)=I_{2}(\mathbf{R}) \delta^{a b}$ with $I_{2}(\mathbf{R})$ the quadratic invariant, so the $\alpha_{i}$ coefficients are simply $I_{2}(\mathbf{R}) / I_{2}(\mathbf{F})=2 I_{2}(\mathbf{R})$ times those in Table II. Similarly, the three-boson diagrams are proportional to

$$
\operatorname{Tr}\left(T_{\mathbf{R}}^{a}\left[T_{\mathbf{R}}^{b}, T_{\mathbf{R}}^{c}\right]\right)=i I_{2}(\mathbf{R}) f^{a b c} .
$$

The fact that both the two and three-boson amplitudes are proportional to the same $I_{2}(\mathbf{R})$ coefficient ensures a proper matching. In particular, the divergence of the three-boson diagrams is correctly accounted for by the $\boldsymbol{\Omega}_{\text {eff }}^{(2)}$ couplings.

The situation is more involved for the four-boson amplitude. The 1PI loops in either the fermion, scalar, or vector case are equivalent two-by-two under the reversing of the loop momentum, so the total amplitudes can always be brought to the form

$$
\begin{aligned}
\mathcal{M}^{a b c d} & =C_{1}^{a b c d} \mathcal{M}_{1}+C_{2}^{a b c d} \mathcal{M}_{2}+C_{3}^{a b c d} \mathcal{M}_{3}, \\
& \left\{\begin{array}{l}
C_{1}^{a b c d}=\operatorname{Tr}\left(T_{\mathbf{R}}^{a} T_{\mathbf{R}}^{b} T_{\mathbf{R}}^{d} T_{\mathbf{R}}^{c}\right)+\operatorname{Tr}\left(T_{\mathbf{R}}^{a} T_{\mathbf{R}}^{c} T_{\mathbf{R}}^{d} T_{\mathbf{R}}^{b}\right), \\
C_{2}^{a b c d}=\operatorname{Tr}\left(T_{\mathbf{R}}^{a} T_{\mathbf{R}}^{b} T_{\mathbf{R}}^{c} T_{\mathbf{R}}^{d}\right)+\operatorname{Tr}\left(T_{\mathbf{R}}^{a} T_{\mathbf{R}}^{d} T_{\mathbf{R}}^{c} T_{\mathbf{R}}^{b}\right), \\
C_{3}^{a b c d}=\operatorname{Tr}\left(T_{\mathbf{R}}^{a} T_{\mathbf{R}}^{c} T_{\mathbf{R}}^{b} T_{\mathbf{R}}^{d}\right)+\operatorname{Tr}\left(T_{\mathbf{R}}^{a} T_{\mathbf{R}}^{d} T_{\mathbf{R}}^{b} T_{\mathbf{R}}^{c}\right),
\end{array}\right.
\end{aligned}
$$

Expanding $\mathcal{M}^{a b c d}$ in the mass of the heavy particle circulating within the loop, only two independent combinations of traces occur at $\mathcal{O}\left(\mathrm{m}^{0}\right)$ and $\mathcal{O}\left(\mathrm{m}^{-2}\right)$, which can be expressed entirely in terms of the quadratic invariants as

$$
\begin{aligned}
D_{1}^{a b c d} & =2 C_{1}^{a b c d}-C_{2}^{a b c d}-C_{3}^{a b c d} \\
& =I_{2}(\mathbf{R})\left(2 f^{a c e} f^{b d e}-f^{a d e} f^{b c e}\right), \\
D_{2}^{a b c d} & =2 C_{2}^{a b c d}-C_{1}^{a b c d}-C_{3}^{a b c d} \\
& =I_{2}(\mathbf{R})\left(2 f^{a d e} f^{b c e}-f^{a c e} f^{b d e}\right), \\
D_{3}^{a b c d} & =2 C_{3}^{a b c d}-C_{1}^{a b c d}-C_{2}^{a b c d} \\
& =I_{2}(\mathbf{R})\left(-f^{a d e} f^{b c e}-f^{a c e} f^{b d e}\right)=-D_{1}^{a b c d}-D_{2}^{a b c d},
\end{aligned}
$$

where we used $\left[T_{\mathbf{R}}^{a}, T_{\mathbf{R}}^{b}\right]=i f^{a b c} T_{\mathbf{R}}^{c}$ together with Eq. (31) and imposed the Jacobi identity $f^{a b e} f^{c d e}=f^{a c e} f^{b d e}$ $f^{a d e} f^{b c e}$. Thanks to this reduction, $\mathcal{M}^{a b c d}$ matches the four-boson amplitude obtained from the $\boldsymbol{Q}_{\text {eff }}^{(2)}$ and $\boldsymbol{R}_{\text {eff }}^{(3)}$ couplings at the $\mathcal{O}\left(\mathrm{m}^{0}\right)$ and $\mathcal{O}\left(\mathrm{m}^{-2}\right)$.

At $\mathcal{O}\left(m^{-4}\right)$, these same combinations $D_{1,2,3}^{a b c d}$ induce the operators tuned by $\gamma_{4,5}$ and $\gamma_{4,6}$, which involve the structure constants. The rest is proportional to the fully symmetrized trace 
$D_{0}^{a b c d}=C_{1}^{a b c d}+C_{2}^{a b c d}+C_{3}^{a b c d}=\frac{1}{4} S \operatorname{Tr}\left(T_{\mathbf{R}}^{a} T_{\mathbf{R}}^{b} T_{\mathbf{R}}^{c} T_{\mathbf{R}}^{d}\right)$.

As detailed in Appendix B, for a general $S U(N)$ algebra, the fully symmetrized trace decomposes into quadratic and quartic invariants. Plugging Eq. (B4) in Eq. (34),

$D_{0}^{a b c d}=6 I_{4}(\mathbf{R}) d^{a b c d}+6 \Lambda(\mathbf{R})\left(\delta^{a b} \delta^{c d}+\delta^{a c} \delta^{b d}+\delta^{a d} \delta^{b c}\right)$,

where $d^{a b c d}$ is the fully symmetric fourth-order symbol normalized such that $I_{4}(\mathbf{F})=1$ for the defining representation, and

$$
\Lambda(\mathbf{R})=\left(\frac{N(\mathbf{A}) I_{2}(\mathbf{R})}{N(\mathbf{R})}-\frac{I_{2}(\mathbf{A})}{6}\right) \frac{I_{2}(\mathbf{R})}{2+N(\mathbf{A})},
$$

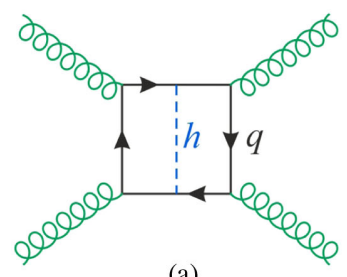

(a)

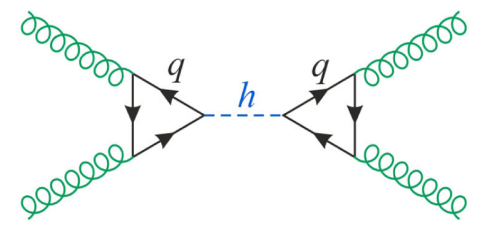

(b)
FIG. 5. Examples of two-loop diagrams in the SM that preserves (a) or violate (b) the one-loop predictions Eq. (38) among the gluonic operators. The particle circulating in the loops are heavy quarks, and the dashed lines denote the Higgs boson.

where A denotes the adjoint representation and $N(\mathbf{R})$ the dimension of the representation $\mathbf{R}$. The term proportional to $\Lambda(\mathbf{R})$ matches onto the operators tuned by $\gamma_{4,1}$ to $\gamma_{4,4}$, while that proportional to $d^{a b c d}$ requires to extend $\boldsymbol{\Omega}_{\text {eff }}^{(4)}$ of Eq. (25) with two extra operators. The total effective Lagrangian is then:

$$
\begin{aligned}
\mathfrak{\Omega}_{\mathrm{eff}}^{(4)}= & \gamma_{4,1} \frac{g_{S}^{4}}{6 ! \pi^{2} m^{4}} G_{\mu \nu}^{a} G^{a, \mu \nu} G_{\rho \sigma}^{b} G^{b, \rho \sigma}+\gamma_{4,2} \frac{g_{S}^{4}}{6 ! \pi^{2} m^{4}} G_{\mu \nu}^{a} \tilde{G}^{a, \mu \nu} G_{\rho \sigma}^{b} \tilde{G}^{b, \rho \sigma}+\gamma_{4,3} \frac{g_{S}^{4}}{6 ! \pi^{2} m^{4}} G_{\mu \nu}^{a} G^{b, \mu \nu} G_{\rho \sigma}^{a} G^{b, \rho \sigma} \\
& +\gamma_{4,4} \frac{g_{S}^{4}}{6 ! \pi^{2} m^{4}} G_{\mu \nu}^{a} \tilde{G}^{b, \mu \nu} G_{\rho \sigma}^{a} \tilde{G}^{b, \rho \sigma}+\gamma_{4,5} \frac{g_{S}^{4}}{6 ! \pi^{2} m^{4}} f^{a b e} f^{c d e} G_{\mu \nu}^{a} G^{c, \mu \nu} G_{\rho \sigma}^{b} G^{d, \rho \sigma}+\gamma_{4,6} \frac{g_{S}^{4}}{6 ! \pi^{2} m^{4}} f^{a b e} f^{c d e} G_{\mu \nu}^{a} \tilde{G}^{c, \mu \nu} G_{\rho \sigma}^{b} \tilde{G}^{d, \rho \sigma} \\
& +\gamma_{4,7} \frac{g_{S}^{4}}{6 ! \pi^{2} m^{4}} d^{a b c d} G_{\mu \nu}^{a} G^{b, \mu \nu} G_{\rho \sigma}^{c} G^{d, \rho \sigma}+\gamma_{4,8} \frac{g_{S}^{4}}{6 ! \pi^{2} m^{4}} d^{a b c d} G_{\mu \nu}^{a} \tilde{G}^{b, \mu \nu} G_{\rho \sigma}^{c} \tilde{G}^{d, \rho \sigma} .
\end{aligned}
$$

The need of a total of eight operators for $S U(N)$ and their connection with the quartic tensor structure is in agreement with Ref. [25]. Note, however, that the definition of $\Lambda(\mathbf{R})$ is a matter of convention, and it indirectly affects the definition of all the operators but those tuned by $\gamma_{4,3}$ and $\gamma_{4,6}$. Yet, adopting the convention in Eq. (36) for $\Lambda(\mathbf{R})$ looks optimal since it ensures $I_{4}(\mathbf{R})=0$ for all $S U(2)$ and $S U(3)$ representations, as it should since these algebras have no irreducible invariant tensor of rank four. As said before, all these results stay valid for $S O(N)$ algebras, but for a single exception. As explained in Appendix B, $S O(8)$ has the unique feature of having two quartic symbols, and an additional term occurs in Eq. (35). In that case, two extra operators are required, tuned by the second quartic symbol of Eq. (B9).

Now, even if a total of eight [or ten for $S O(8)$ ] independent operators can be constructed in general, our specific computations show that at one loop, most of these operators derive from a single symmetrized trace and are thus always correlated. In particular, no matter the representation or spin of the particle in the loop:

$$
\gamma_{4,1}=\frac{1}{2} \gamma_{4,3}
$$

$$
\gamma_{4,2}=\frac{1}{2} \gamma_{4,4}
$$

There are thus two operator combinations that never occur in the one-loop effective action. From an effective theory point of view, this should remain true in most cases since it derives from the symmetry of the amplitude. A necessary condition beyond one-loop is the absence of diagrams where the color flow is disconnected, that is, where a product of traces over the generators occurs instead of a single trace. This never happens if only one heavy state is integrated out, but could arise in more general settings. For example, in the SM, integrating out heavy quarks together with the Higgs boson, the diagrams in Fig. 5 arise at two loops. Since the $C P$-conserving effective Higgs coupling to two gluons is of the form $h^{0} G_{\mu \nu}^{a} G^{a, \mu \nu}$, it is clear that the Higgs boson exchange in Fig. 5(b) contribute to $\gamma_{4,1}$ but not to $\gamma_{4,3}$.

The coefficients for a complex field (fermion, scalar, vector particle) circulating in the loops are given in Table III. Those for a self-conjugate particle are half of those quoted there. Indeed, when the propagator is not oriented, some Feynman diagrams get an extra symmetry factor $1 / 2$, while for others, the loop momentum cannot be 
TABLE III. Wilson coefficients of the effective operators for $S U(N)$ or $S O(N \neq 8)$ gauge bosons, as induced by a set of complex fields of spin $0,1 / 2$, and 1 transforming under the representation $\mathbf{R}$. For real fields, all the coefficients should be halved.

\begin{tabular}{lcccccc}
\hline \hline & $\alpha_{0}$ & $\alpha_{2}$ & $\alpha_{4}$ & $\beta_{2}$ & $\beta_{4,1}$ & $\beta_{4,2}$ \\
\hline Scalar & $\frac{1}{2} I_{2}(\mathbf{R}) D_{\varepsilon}$ & $-\frac{1}{8} I_{2}(\mathbf{R})$ & $\frac{3}{56} I_{2}(\mathbf{R})$ & $\frac{1}{24} I_{2}(\mathbf{R})$ & $-\frac{1}{14} I_{2}(\mathbf{R})$ & 0 \\
Fermion & $2 I_{2}(\mathbf{R}) D_{\varepsilon}$ & $-I_{2}(\mathbf{R})$ & $\frac{9}{14} I_{2}(\mathbf{R})$ & $-\frac{1}{12} I_{2}(\mathbf{R})$ & $\frac{1}{7} I_{2}(\mathbf{R})$ & $-\frac{3}{2} I_{2}(\mathbf{R})$ \\
Vector & $-\frac{21 D_{\varepsilon}+2}{2} I_{2}(\mathbf{R})$ & $\frac{37}{8} I_{2}(\mathbf{R})$ & $-\frac{159}{56} I_{2}(\mathbf{R})$ & $\frac{1}{8} I_{2}(\mathbf{R})$ & $-\frac{3}{14} I_{2}(\mathbf{R})$ & $6 I_{2}(\mathbf{R})$ \\
\hline \hline & $\gamma_{4,1}=\gamma_{4,3} / 2$ & $\gamma_{4,2}=\gamma_{4,4} / 2$ & $\gamma_{4,5}$ & $\gamma_{4,6}$ & $\gamma_{4,7}$ & $\gamma_{4,8}$ \\
\hline Scalar & $\frac{7}{32} \Lambda(\mathbf{R})$ & $\frac{1}{32} \Lambda(\mathbf{R})$ & $\frac{1}{48} I_{2}(\mathbf{R})$ & $\frac{1}{336} I_{2}(\mathbf{R})$ & $\frac{7}{32} I_{4}(\mathbf{R})$ & $\frac{1}{32} I_{4}(\mathbf{R})$ \\
Fermion & $\frac{1}{2} \Lambda(\mathbf{R})$ & $\frac{7}{8} \Lambda(\mathbf{R})$ & $\frac{1}{48} I_{2}(\mathbf{R})$ & $\frac{19}{336} I_{2}(\mathbf{R})$ & $\frac{1}{2} I_{4}(\mathbf{R})$ & $\frac{7}{8} I_{4}(\mathbf{R})$ \\
Vector & $\frac{261}{32} \Lambda(\mathbf{R})$ & $\frac{243}{32} \Lambda(\mathbf{R})$ & $-\frac{3}{16} I_{2}(\mathbf{R})$ & $-\frac{27}{112} I_{2}(\mathbf{R})$ & $\frac{261}{32} I_{4}(\mathbf{R})$ & $\frac{243}{32} I_{4}(\mathbf{R})$ \\
\hline \hline
\end{tabular}

reversed and runs in only one direction. This latter situation also brings a factor $1 / 2$ because $\left(T_{\mathbf{R}}^{a}\right)^{T}=-T_{\mathbf{R}}^{a}$ for a real representation. For example, instead of Eq. (31), the triangle diagrams are now tuned by

$$
\begin{aligned}
\left.\operatorname{Tr}\left(T_{\mathbf{R}}^{a} T_{\mathbf{R}}^{b} T_{\mathbf{R}}^{c}\right)\right|_{\text {self-conjugate }} & =\frac{1}{2} \operatorname{Tr}\left(T_{\mathbf{R}}^{a}\left[T_{\mathbf{R}}^{b}, T_{\mathbf{R}}^{c}\right]\right) \\
& =\frac{1}{2} i I_{2}(\mathbf{R}) f^{a b c} .
\end{aligned}
$$

Similarly, the coefficients for the four-point amplitude satisfy

$$
\begin{aligned}
\left.C_{1}^{a b c d}\right|_{\text {self-conjugate }} & =\operatorname{Tr}\left(T_{\mathbf{R}}^{a} T_{\mathbf{R}}^{b} T_{\mathbf{R}}^{d} T_{\mathbf{R}}^{c}\right) \\
& =\frac{1}{2}\left(\operatorname{Tr}\left(T_{\mathbf{R}}^{a} T_{\mathbf{R}}^{c} T_{\mathbf{R}}^{d} T_{\mathbf{R}}^{b}\right)+\operatorname{Tr}\left(T_{\mathbf{R}}^{a} T_{\mathbf{R}}^{c} T_{\mathbf{R}}^{d} T_{\mathbf{R}}^{b}\right)\right) \\
& =\frac{1}{2} C_{1}^{a b c d}
\end{aligned}
$$

We checked this property of the coefficients for two physically relevant cases: the contribution to the gluon coefficients of the $S U(5)$ Higgs bosons $H_{G}^{a}$ and of the

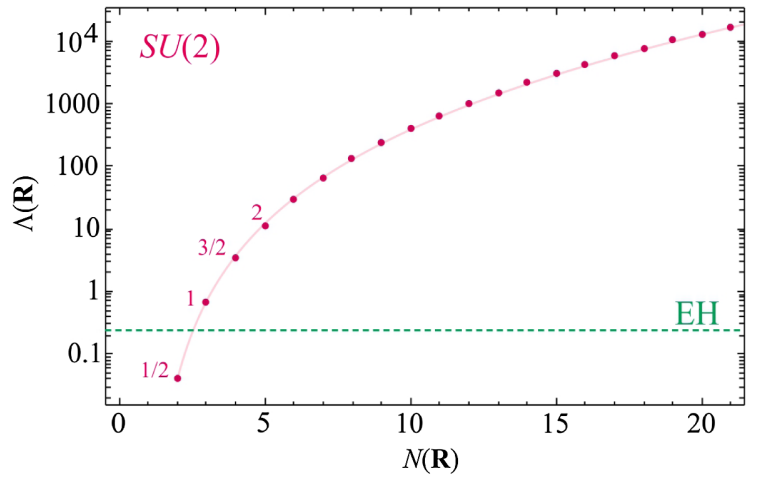

MSSM gluinos, both self-conjugate fields transforming in the adjoint representation of $S U(3)_{C}$.

\section{A. Reduction to $S U(3)$ and $S U(2)$}

The general basis of effective operators reduces immediately to $S U(3)$ by removing the quartic invariant operators, i.e., by setting $\gamma_{4,7}$ and $\gamma_{4,8}$ to zero. For the fundamental representation, $I_{2}^{S U(3)}(\mathbf{F})=1 / 2$ and $\Lambda^{S U(3)}(\mathbf{F})=1 / 24$, and we recover the results of Table II. But, an interesting feature appears for more general representations. A priori, as the representation get larger, one would expect the strength of the effective interactions to increase mechanically due to the increased number of particles circulating in the loop. However, we show in Fig. 6 that $\Lambda(\mathbf{R})$ grows much faster than $N(\mathbf{R})$. The fastest growth happens for representations which are the symmetric tensor products of the fundamental representations, for which $\Lambda(\mathbf{R}) \sim N(\mathbf{R})^{3}$. For instance, $\Lambda(\mathbf{3})=1 / 24$ but $\Lambda\left(\mathbf{6}=\mathbf{3} \otimes_{S} \mathbf{3}\right)=17 / 24$, $\Lambda\left(\mathbf{1 0}=\mathbf{3} \otimes_{S} \mathbf{3} \otimes_{S} \mathbf{3}\right)=99 / 24$, and $\Lambda\left(\mathbf{1 5}=\mathbf{3} \otimes_{S} \mathbf{3} \otimes_{S} \mathbf{3} \otimes_{S} \mathbf{3}\right)=$ $371 / 24$. The adjoint representation is not on this series, but the effective interactions are nevertheless stronger than

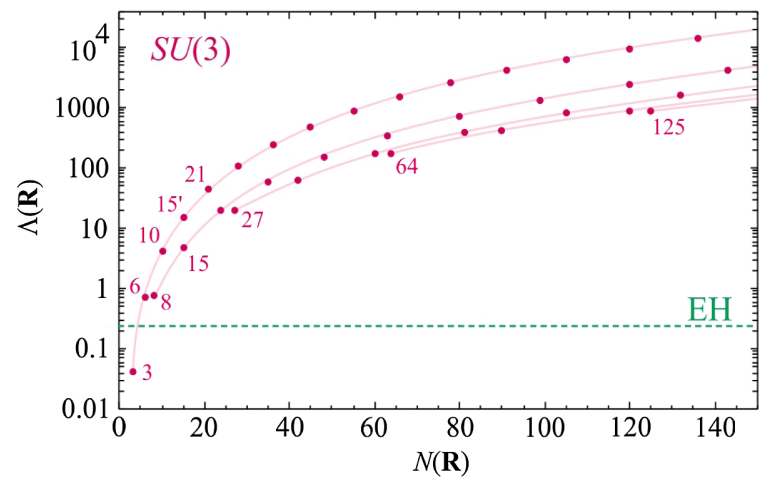

FIG. 6. Evolution of $\Lambda(\mathbf{R})$ as a function of the dimension $N(\mathbf{R})$ for $S U(2)$ and $S U(3)$. In the former case, we denote the first few representations by the corresponding isospin. In the $S U(3)$ case, several branches are apparent, each starting with a real representation. The horizontal dashed lines depict the Euler-Heisenberg value, identified as $\Lambda(\mathbf{1})=1 / 3$ for a charge-one loop particle from Eq. (48). 
naively expected from the dimension since $\Lambda^{S U(3)}(\mathbf{8})=$ $3 / 4=18 \times \Lambda^{S U(3)}(\mathbf{3})$. Interestingly, this corresponds to physically sensible scenarios, for example that of the gluinos in the MSSM for which (including the 1/2 factor for self-conjugate particles):

$$
\frac{1}{2} \times \frac{g_{S}^{4}}{6 ! \pi^{2} m_{\tilde{g}}^{4}} \gamma_{4,1}=-\frac{1}{2} \times \frac{1}{2} 18 \frac{g_{S}^{4}}{6 ! \pi^{2} m_{\tilde{g}}^{4}}=\frac{\alpha_{S}}{10 m_{\tilde{g}}^{4}},
$$

which is an order of magnitude larger than the coefficient of the effective photon interactions of the Euler-Heisenberg Lagrangian.

For $S U(2)$, the effective Lagrangian gets simpler thanks to the identity

$$
f^{a b e} f^{c d e} \rightarrow \varepsilon^{a b e} \varepsilon^{c d e}=\delta^{a c} \delta^{b d}-\delta^{a d} \delta^{b c},
$$

which permits us to get rid of two operators. Expressing the remaining four operators explicitly in terms of the $S U(2)$ triplet states denoted as $\left\{W_{\mu}^{-}, W_{\mu}^{3}, W_{\mu}^{+}\right\}$:

$$
\begin{aligned}
\mathfrak{\Omega}_{\mathrm{eff}, S U(2)_{L}}^{(4)}= & \frac{\left(\gamma_{4,1}+\gamma_{4,3}\right) g^{4}}{6 ! \pi^{2} m^{4}}\left(W_{\mu \nu}^{3} W^{3, \mu \nu}\right)^{2}+\frac{\left(\gamma_{4,2}+\gamma_{4,4}\right) g^{4}}{6 ! \pi^{2} m^{4}}\left(W_{\mu \nu}^{3} \tilde{W}^{3, \mu \nu}\right)^{2}+\frac{4\left(\gamma_{4,1}+\gamma_{4,5}\right) g^{4}}{6 ! \pi^{2} m^{4}} W_{\mu \nu}^{3} W^{3, \mu \nu} W_{\rho \sigma}^{+} W^{-, \rho \sigma} \\
& +\frac{4\left(\gamma_{4,2}+\gamma_{4,6}\right) g^{4}}{6 ! \pi^{2} m^{4}} W_{\mu \nu}^{3} \tilde{W}^{3, \mu \nu} W_{\rho \sigma}^{+} \tilde{W}^{-, \rho \sigma}+\frac{4\left(\gamma_{4,3}-\gamma_{4,5}\right) g^{4}}{6 ! \pi^{2} m^{4}}\left|W_{\mu \nu}^{3} W^{+, \mu \nu}\right|^{2}+\frac{4\left(\gamma_{4,4}-\gamma_{4,6}\right) g^{4}}{6 ! \pi^{2} m^{4}}\left|W_{\mu \nu}^{3} \tilde{W}^{+, \mu \nu}\right|^{2} \\
& +\frac{2\left(2 \gamma_{4,1}+\gamma_{4,3}+\gamma_{4,5}\right) g^{4}}{6 ! \pi^{2} m^{4}}\left(W_{\mu \nu}^{+} W^{-, \mu \nu}\right)^{2}+\frac{2\left(\gamma_{4,4}-\gamma_{4,6}\right) g^{4}}{6 ! \pi^{2} m^{4}}\left|W_{\mu \nu}^{+} \tilde{W}^{+, \mu \nu}\right|^{2} \\
& +\frac{2\left(\gamma_{4,3}-\gamma_{4,5}\right) g^{4}}{6 ! \pi^{2} m^{4}}\left|W_{\mu \nu}^{+} W^{+, \mu \nu}\right|^{2}+\frac{2\left(2 \gamma_{4,2}+\gamma_{4,4}+\gamma_{4,6}\right) g^{4}}{6 ! \pi^{2} m^{4}}\left(W_{\mu \nu}^{+} \tilde{W}^{-, \mu \nu}\right)^{2} .
\end{aligned}
$$

These operators and coefficients are obtained from the effective action, and are independent of the invariant mass of the external states. Thus, they remain valid for massive external weak bosons, at least as long as $m$ is sufficiently large compared to $M_{Z, W}$. An important caveat though, of relevance for the SM, is the presence of chiral fermions. Those cannot be massive without breaking the gauge symmetry, so the inverse mass expansion is defined only in the broken phase. Nongauge invariant operators can then arise, at both the $\mathcal{O}\left(\mathrm{m}^{0}\right)$ and $\mathcal{O}\left(\mathrm{m}^{-2}\right)$ level.

Concerning the strength of the effective interactions, here also $\Lambda(\mathbf{R})$ grows much faster than $N(\mathbf{R})$. Actually, as the $S U(2)$ representations are smaller than those of $S U(3)$, the increase is much more pronounced, with $\Lambda(\mathbf{R}) \sim N(\mathbf{R})^{5}$, see Fig. 6 . So, while $\Lambda(\mathbf{F})=1 / 24$, it is already an order of magnitude stronger for the adjoint representation, $\Lambda(\mathbf{3})=2 / 3=16 \times \Lambda(\mathbf{2})$.

To close this section, it is instructive to look at the application of the $S U(N)$ result from a group-theoretic perspective. Up to now, the $S U(2)$ and $S U(3)$ effective Lagrangians are obtained simply by setting $N=2$ or $N=3$ in the general result. But, if $S U(N)$ is large enough to contain an $S U(2)$ or $S U(3)$ subalgebra, we could also ask where these pieces are in the general $S U(N)$ Lagrangian. More generally, consider the effective Lagrangian for a representation $\mathbf{R}_{M}$ of $S U(M)$. These $N\left(\mathbf{R}_{M}\right)$ states organize themselves into representations of $S U(N) \subset S U(M)$, that is, $\mathbf{R}_{M}$ branches into a direct sum of $S U(N)$ representations $\mathbf{R}_{N}$. So, from the $S U(N)$ perspective, the $S U(M)$ coefficients encode the circulation of a collection of states in the loop. Since these contributions simply add up, the $S U(M)$ coefficients must be the sum over the $S U(N)$ coefficients for all the $\mathbf{R}_{N}$ representations present in the representation $\mathbf{R}_{M}$. Going back to Eq. (35), we must thus have

$$
\begin{aligned}
\frac{1}{6} D_{0}^{a b c d} & =I_{4}\left(\mathbf{R}_{M}\right) d_{M}^{a b c d}+\Lambda_{N}\left(\mathbf{R}_{M}\right)\left(\delta^{a b} \delta^{c d}+\delta^{a c} \delta^{b d}+\delta^{a d} \delta^{b c}\right) \\
& =\sum_{\mathbf{R}_{N} \subset \mathbf{R}_{M}} I_{4}\left(\mathbf{R}_{N}\right) d_{N}^{a b c d} \\
& +\sum_{\mathbf{R}_{N} \subset \mathbf{R}_{M}} \Lambda_{N}\left(\mathbf{R}_{N}\right)\left(\delta^{a b} \delta^{c d}+\delta^{a c} \delta^{b d}+\delta^{a d} \delta^{b c}\right),
\end{aligned}
$$

where the indices $a, b, c, d$ are understood to denote those $S U(M)$ generators that correspond to the $S U(N)$ subalgebra. The main difficulty though is that even restricted to those particular generators, $d_{M}^{a b c d} \neq d_{N}^{a b c d}$ because the definition of the quartic invariant involves different functions $\Lambda_{N}$ and $\Lambda_{M}$. To proceed, let us assume that the fundamental representation has the branching rule $\mathbf{F}_{M} \rightarrow \mathbf{F}_{N}$. Knowing that by definition, $I_{4}\left(\mathbf{F}_{M}\right)=I_{4}\left(\mathbf{F}_{N}\right)=1$, we find

$$
\begin{array}{r}
I_{4}\left(\mathbf{R}_{M}\right)=\sum_{\mathbf{R}_{N} \subset \mathbf{R}_{M}} I_{4}\left(\mathbf{R}_{N}\right), \\
I_{4}\left(\mathbf{R}_{M}\right)\left(\Lambda_{N}\left(\mathbf{F}_{N}\right)-\Lambda_{M}\left(\mathbf{F}_{M}\right)\right)+\Lambda_{M}\left(\mathbf{R}_{M}\right)=\sum_{\mathbf{R}_{N} \subset \mathbf{R}_{M}} \Lambda_{N}\left(\mathbf{R}_{N}\right) .
\end{array}
$$

Using the numbers quoted in Appendix B and the branching rules in Ref. [60], one can check that the two formulas are valid for $S U(3) \subset S U(4)$ and $S U(4) \subset S U(5)$. The second one also applies to $S U(2) \subset S U(3)$ in which case it becomes a sum rule for the $\Lambda$ functions since $I_{4}(\mathbf{R})=0$ in $S U(3)$. 
From a calculation point of view, once the branching rules of the $S U(M)$ representations are known, these equations are particularly powerful, with the second one even allowing to compute $I_{4}\left(\mathbf{R}_{M}\right)$ in terms of $\Lambda_{N}$ and $\Lambda_{M}$, that is, entirely in terms of the quadratic invariants $I_{2}\left(\mathbf{R}_{N}\right)$ and $I_{2}\left(\mathbf{R}_{M}\right)$.

Thanks to the convention Eq. (36), the branching rule for the $I_{4}$ invariant is very simple [34], but there is a price to pay. Some part of the $\gamma_{4,7}$ and $\gamma_{4,8}$ operators of $S U(M)$ are moved into the $\gamma_{4,1}$ to $\gamma_{4,4}$ operators of $S U(N<M)$. This is due to the very definition of the operators in terms of different quartic symbols, and not related to the loop structure of the amplitude or the specific branching rules. For example, if for some unification group a specific mechanism is found that generates only $\gamma_{4,7}$ and $\gamma_{4,8}$, the four operators tuned by $\gamma_{4,1}$ to $\gamma_{4,4}$ are in general present once the symmetry is spontaneously broken simply because the $d^{a b c d}$ symbol is defined differently within the surviving subalgebra.

\section{B. Reduction to $\boldsymbol{U}(\mathbf{1})$}

Comparing the $S U(N)$ coefficients $\gamma_{4, i}$ in Table III with the Euler-Heisenberg results in Table I, the two clearly appear related. Heuristically, it is simple to understand this relationship by adapting the decomposition Eq. (32) to the $U(1)$ case. When only a single generator occurs, $C_{1}=C_{2}=C_{3}=2 Q^{4}$. This ensures the cancellation of the UV divergence, and more generally the absence of all the operators tuned by the structure constants. The whole amplitude is then proportional to

$$
D_{0} \equiv C_{1}+C_{2}+C_{3}=6 Q^{4} .
$$

Since the same factor of 6 occurs in the $S U(N)$ result in Eq. (35), it is clear that $\gamma_{4,1}^{\mathrm{EH}}$ and $\gamma_{4,2}^{\mathrm{EH}}$ can be obtained equivalently from $\gamma_{4,1}^{S U(N)}, \gamma_{4,2}^{S U(N)}$ with $\Lambda(\mathbf{R}) \rightarrow Q^{4}$ or from $\gamma_{4,7}^{S U(N)}, \quad \gamma_{4,8}^{S U(N)}$ with $I_{4}(\mathbf{R}) \rightarrow Q^{4}$, in agreement with Tables III and I. Obviously, this line of reasoning is a naive identification of the coefficients of the loop functions, not a group-theoretic reduction of $S U(N)$ down to one of its $U(1)$ subgroup.

To perform a true reduction, let us denote $T^{a}$ one of the diagonal generators of the Cartan algebra of $S U(N)$. This generator induces a $U(1)_{\alpha} \subset S U(N)$ for which the $S U(N)$ effective Lagrangian reduces to

$$
\begin{aligned}
\mathfrak{Q}_{\mathrm{eff}}^{(4)} & \left(U(1)_{\alpha} \subset S U(N)\right) \\
= & \left(\gamma_{4,1}+\gamma_{4,3}+d^{\alpha \alpha \alpha \alpha} \gamma_{4,7}\right) \frac{g_{S}^{4}}{6 ! \pi^{2} m^{4}} G_{\mu \nu}^{\alpha} G^{\alpha, \mu \nu} G_{\rho \sigma}^{\alpha} G^{\alpha, \rho \sigma} \\
& \quad+\left(\gamma_{4,2}+\gamma_{4,4}+d^{\alpha \alpha \alpha \alpha} \gamma_{4,8}\right) \frac{g_{S}^{4}}{6 ! \pi^{2} m^{4}} G_{\mu \nu}^{\alpha} \tilde{G}^{\alpha, \mu \nu} G_{\rho \sigma}^{\alpha} \tilde{G}^{\alpha, \rho \sigma} .
\end{aligned}
$$

The Euler-Heisenberg result must arise from a combination of six of the eight $S U(N)$ operators, including those involving the quartic invariant. Looking back at their values in Table III for a given representation $\mathbf{R}$, this reduction matches the results in Table I for scalar, fermion, and vector provided a single condition is satisfied:

$$
3 \Lambda(\mathbf{R})+d^{\alpha \alpha \alpha \alpha} I_{4}(\mathbf{R})=\sum_{q_{\alpha} \in \mathbf{R}} q_{\alpha}^{4} .
$$

The sum on the right-hand side is carried over all the states in the representation $\mathbf{R}$. To see that this condition holds in general, it suffices to go back to the very definition of the quartic invariant, Eq. (B4), which becomes for a single generator:

$\frac{1}{4 !} S \operatorname{Tr}\left(T_{\mathbf{R}}^{\alpha} T_{\mathbf{R}}^{\alpha} T_{\mathbf{R}}^{\alpha} T_{\mathbf{R}}^{\alpha}\right)=\operatorname{Tr}\left(\left(T_{\mathbf{R}}^{\alpha}\right)^{4}\right)=I_{4}(\mathbf{R}) d^{\alpha \alpha \alpha \alpha}+3 \Lambda(\mathbf{R})$.

Since $T_{\mathbf{R}}^{\alpha}$ is diagonal, the trace collapses to a sum over the quartic power of its eigenvalues, i.e., over the quartic power of the $U(1)_{\alpha}$ charges of the states of the representation $\mathbf{R}$. The final step to match Table I is to rescale the generator $T_{\mathbf{R}}^{\alpha}$ to properly normalize the $U(1)_{\alpha}$ charge in units of $Q$. Note that this relation can be trivially generalized to other Casimir invariants. In particular, for the dimension-four and six operators, $I_{2}(\mathbf{R})=\operatorname{Tr}\left(\left(T_{\mathbf{R}}^{\alpha}\right)^{2}\right)=\sum_{q_{\alpha} \in \mathbf{R}} q_{\alpha}^{2}$, showing that the $\alpha_{i}$ coefficients for $S U(N)$ reduce to those for QED under the naive substitution $I_{2}(\mathbf{R}) \rightarrow Q^{2}$ in Table III.

Numerical applications to illustrate this formula are in Appendix B. Note that for both $S U(2)$ and $S U(3)$, there is no quartic invariant and the Euler-Heisenberg coefficients for a single unit charge state are formally obtained setting $\Lambda(\mathbf{1})=1 / 3$ in Eq. (48). This value is plotted in Fig. 6 for comparison.

\section{Reduction to factor groups}

The general result also reduces to mixed interactions, involving the gauge bosons of two different algebras. Before investigating this reduction, let us directly compute them using FeynArTs models. For that, we consider the photon-gluon interactions induced by quark, squark, or $S U(5)$ leptoquark loops in the nonlinear gauge (see Fig. 7). It is then a simple matter to generalize the results obtained for the fundamental $S U(3)_{C}$ representation to that for generic $S U(N)$ representations. The loops are finite and the effective interactions start at the dimension-eight level,

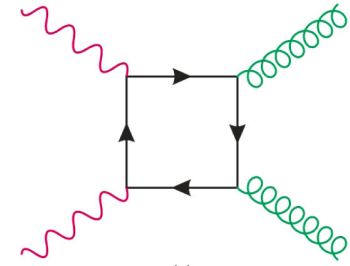

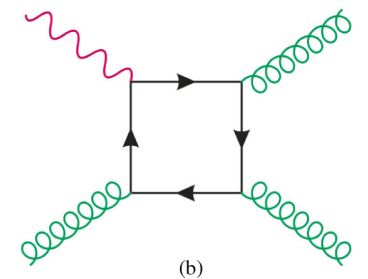

(b)
FIG. 7. Quark loops generating the effective dimension-eight photon-gluon interactions. 


$$
\begin{aligned}
\mathfrak{Q}_{\mathrm{eff}}^{(4)}(U(1) \otimes S U(N))= & \alpha_{1} \frac{g_{1}^{2} g_{n}^{2}}{6 ! \pi^{2} m^{4}} F_{\mu \nu} F^{\mu \nu} G_{\rho \sigma}^{a} G^{a, \rho \sigma}+\alpha_{2} \frac{g_{1}^{2} g_{n}^{2}}{6 ! \pi^{2} m^{4}} F_{\mu \nu} \tilde{F}^{\mu \nu} G_{\rho \sigma}^{a} \tilde{G}^{a, \rho \sigma}+\alpha_{3} \frac{g_{1}^{2} g_{n}^{2}}{6 ! \pi^{2} m^{4}} F_{\mu \nu} G^{a, \mu \nu} F_{\rho \sigma} G^{a, \rho \sigma} \\
& +\alpha_{4} \frac{g_{1}^{2} g_{n}^{2}}{6 ! \pi^{2} m^{4}} F_{\mu \nu} \tilde{G}^{a, \mu \nu} F_{\rho \sigma} \tilde{G}^{a, \rho \sigma}+\beta_{1} \frac{g_{1} g_{n}^{3}}{6 ! \pi^{2} m^{4}} d^{a b c} F_{\mu \nu} G^{a, \mu \nu} G_{\rho \sigma}^{b} G^{c, \rho \sigma} \\
& +\beta_{2} \frac{g_{1} g_{n}^{3}}{6 ! \pi^{2} m^{4}} d^{a b c} F_{\mu \nu} \tilde{G}^{a, \mu \nu} G_{\rho \sigma}^{b} \tilde{G}^{c, \rho \sigma}
\end{aligned}
$$

where $g_{1}$ and $g_{n}$ denote the $U(1)$ and $S U(N)$ coupling constants, respectively. The numerical values of the Wilson coefficients are in Table IV. They are invariant under charge conjugation since $Q\left(\mathbf{R}^{*}\right)=-Q(\mathbf{R}), I_{2}\left(\mathbf{R}^{*}\right)=+I_{2}(\mathbf{R})$, and $I_{3}\left(\mathbf{R}^{*}\right)=-I_{3}(\mathbf{R})$, and they obviously vanish for a real representation. Note in particular that the $S U(5)$ leptoquarks give $\beta_{i}<0$ since the electric charge of the antitriplet is positive, $Q(\overline{\mathbf{3}})=+\sqrt{5 / 12}$.

The first four interactions are immediately extended to the case of two $S U(N)$ and two $S U(M)$ gauge bosons. Specifically, the operators are then

$$
\begin{aligned}
\mathbf{\Omega}_{\mathrm{eff}}^{(4)}(S U(M) \otimes S U(N))= & \alpha_{1} \frac{g_{m}^{2} g_{n}^{2}}{6 ! \pi^{2} m^{4}} W_{\mu \nu}^{i} W^{i, \mu \nu} G_{\rho \sigma}^{a} G^{a, \rho \sigma}+\alpha_{2} \frac{g_{m}^{2} g_{n}^{2}}{6 ! \pi^{2} m^{4}} W_{\mu \nu}^{i} \tilde{W}^{i, \mu \nu} G_{\rho \sigma}^{a} \tilde{G}^{a, \rho \sigma} \\
& +\alpha_{3} \frac{g_{m}^{2} g_{n}^{2}}{6 ! \pi^{2} m^{4}} W_{\mu \nu}^{i} G^{a, \mu \nu} W_{\rho \sigma}^{i} G^{a, \rho \sigma}+\alpha_{4} \frac{g_{m}^{2} g_{n}^{2}}{6 ! \pi^{2} m^{4}} W_{\mu \nu}^{i} \tilde{G}^{a, \mu \nu} W_{\rho \sigma}^{i} \tilde{G}^{a, \rho \sigma},
\end{aligned}
$$

where $g_{m}$ and $g_{n}$ denote the $S U(M)$ and $S U(N)$ coupling constants, respectively. Looking at Fig. 7(a), it is easy to realize that the coefficients are obtained from those for $U(1)$ in Table IV by replacing $Q(\mathbf{R})^{2} I_{2}(\mathbf{R}) \rightarrow$ $I_{2}^{M}\left(\mathbf{R}_{M}\right) I_{2}^{N}\left(\mathbf{R}_{N}\right)$ when the particles in the loop are in the $\left(\mathbf{R}_{M}, \mathbf{R}_{N}\right)$ representation of $S U(M) \otimes S U(N)$.

For the SM, the case $S U(2)_{L} \otimes S U(3)_{C}$ is immediately obtained in the $\left\{W_{\mu}^{-}, W_{\mu}^{3}, W_{\mu}^{+}\right\}$basis by replacing $W_{\mu \nu}^{i} W^{i, \mu \nu}=W_{\mu \nu}^{3} W^{3, \mu \nu}+2 W_{\mu \nu}^{+} W^{-, \mu \nu}$ and $g_{n} \rightarrow g, g_{m} \rightarrow g_{S}$. Note however that the same caveat as for the effective interactions in Eq. (43) applies. In the presence of chiral fermions, these interactions are not leading and dimensionsix operators of $\mathcal{O}\left(m^{-2}\right)$ appear, like e.g., $\tilde{G}_{\mu \nu}^{a} G^{a, \nu \rho} Z^{\mu \rho}$ or $Z_{\mu} Z_{\rho} G_{\mu \nu}^{a} G^{a, \rho \nu}$ inducing $Z \rightarrow g g g$ [61] and $g g \rightarrow Z Z$ [62]. The only exceptions are the $Z \rightarrow g g \gamma$ [63] and $Z \rightarrow \gamma \gamma \gamma$ [64] interactions for on shell gluons and photons, which still start at $\mathcal{O}\left(\mathrm{m}^{-4}\right)$ for chiral fermions because the $\gamma_{5}$ term of the $Z$ boson coupling to fermions cancels out. On shell, these effective interactions are simply obtained from the $\gamma \gamma \rightarrow g g$ and $\gamma \gamma \rightarrow \gamma \gamma$ results by rescaling of one photon couplings to match that of the $Z$ boson.

Because $U(1) \otimes S U(N) \subset S U(M \geq N+1)$, the $\alpha_{i}, \beta_{i}$ coefficients in Table IV are directly related to the $\gamma_{4, i}$ in Table III, which is not very surprising comparing their values. As for the reduction down to $U(1)$ in the previous section, this can be understood looking at the coefficients of the loop functions. For the $\alpha_{i}$ coefficients, the decomposition Eq. (32) becomes $C_{1}^{a b}=C_{2}^{a b}=C_{3}^{a b}=2 I_{2}(\mathbf{R}) Q^{2} \delta^{a b}$, hence $D_{0}^{a b}=6 I_{2}(\mathbf{R}) Q^{2} \delta^{a b}$. Comparing with Eq. (35), we see that $\alpha_{i}=2 \gamma_{4, i}$ with the replacement $\Lambda\left(\mathbf{R}_{M}\right) \rightarrow$ $Q\left(\mathbf{R}_{N}\right)^{2} I_{2}\left(\mathbf{R}_{N}\right)$ in Table III. The factor of two comes from the two ways of identifying the $U(1)$ and $S U(N)$ gauge bosons, e.g., $\left(G_{\mu \nu}^{a} G^{a, \mu \nu}\right)_{M}^{2} \rightarrow 2\left(F_{\rho \sigma} F^{\rho \sigma}\right)\left(G_{\mu \nu}^{a} G^{a, \mu \nu}\right)_{N}$. A similar reasoning can be done for the $\beta_{i}$ coefficients.

To go beyond a naive identification of the loop functions, let us denote $T^{a}$ the Cartan generator of $S U(M)$ generating $U(1)$ and $T^{i}, i=2, \ldots, N^{2}-1$ those generating $S U(N)$. Because $\left[T^{\alpha}, T^{i}\right]=0$ implies $f^{\alpha i a}=0$, the UV divergent

TABLE IV. Wilson coefficients of the effective operators for the mixed operators, as induced by a complex field (scalar, fermion, vector boson) in the representation $\mathbf{R}$ of $S U(N)$ with $U(1)$ charge $Q(\mathbf{R})$. The $\alpha_{i}$ coefficients for two $S U(N)$ and two $S U(M)$ gauge bosons are obtained by replacing $Q(\mathbf{R})^{2} I_{2}(\mathbf{R}) \rightarrow I_{2}^{M}\left(\mathbf{R}_{M}\right) I_{2}^{N}\left(\mathbf{R}_{N}\right)$.

\begin{tabular}{lcccc}
\hline \hline & $\alpha_{1}=\alpha_{3} / 2$ & $\alpha_{2}=\alpha_{4} / 2$ & $\beta_{1}$ & $\beta_{2}$ \\
\hline Scalar & $\frac{7}{16} Q(\mathbf{R})^{2} I_{2}(\mathbf{R})$ & $\frac{1}{16} Q(\mathbf{R})^{2} I_{2}(\mathbf{R})$ & $\frac{7}{32} Q(\mathbf{R}) I_{3}(\mathbf{R})$ & $\frac{1}{32} Q(\mathbf{R}) I_{3}(\mathbf{R})$ \\
Fermion & $Q(\mathbf{R})^{2} I_{2}(\mathbf{R})$ & $\frac{7}{4} Q(\mathbf{R})^{2} I_{2}(\mathbf{R})$ & $\frac{1}{2} Q(\mathbf{R}) I_{3}(\mathbf{R})$ & $\frac{7}{8} Q(\mathbf{R}) I_{3}(\mathbf{R})$ \\
Vector & $\frac{261}{16} Q(\mathbf{R})^{2} I_{2}(\mathbf{R})$ & $\frac{243}{16} Q(\mathbf{R})^{2} I_{2}(\mathbf{R})$ & $\frac{261}{32} Q(\mathbf{R}) I_{3}(\mathbf{R})$ & $\frac{243}{32} Q(\mathbf{R}) I_{3}(\mathbf{R})$ \\
\hline \hline
\end{tabular}


contributions disappear and the $\gamma_{4,5}^{S U(M)}$ and $\gamma_{4,6}^{S U(M)}$ operators do not contribute to the $U(1) \otimes S U(N)$ effective operators. For the other coefficients, consider a specific representation of $S U(M)$ with branching rule $\mathbf{R}_{M} \rightarrow \sum \mathbf{R}_{N}$, and denote $q_{\alpha}\left(\mathbf{R}_{N}\right)$ the $U(1)_{\alpha}$ charge of the states of the representation $\mathbf{R}_{N}$. Mathematically, this branching rule means $N^{2}$ of the $T_{\mathbf{R}_{M}}$ generators of $S U(M)$ can be brought to a block diagonal form. Those corresponding to $S U(N)$ have blocks containing the $S U(N)$ generators in the representation $\mathbf{R}_{N}$, while the $T^{\alpha}$ generator is a diagonal matrix containing all the $q_{\alpha}\left(\mathbf{R}_{N}\right)$ charges, which are constant over each block since $\left[T^{\alpha}, T^{i}\right]=0$. The fully symmetrized trace with two or three $S U(N)$ generators then necessarily take the form

$$
\begin{aligned}
\frac{1}{4 !} S \operatorname{Tr}\left(T_{\mathbf{R}}^{\alpha} T_{\mathbf{R}}^{\alpha} T_{\mathbf{R}}^{i} T_{\mathbf{R}}^{j}\right) & =\Lambda\left(\mathbf{R}_{M}\right) \delta^{i j}+d^{\alpha \alpha i j} I_{4}\left(\mathbf{R}_{M}\right) \\
& =\sum_{\mathbf{R}_{N} \subset \mathbf{R}_{M}} q_{\alpha}\left(\mathbf{R}_{N}\right)^{2} I_{2}\left(\mathbf{R}_{N}\right) \delta^{i j}, \\
\frac{1}{4 !} S \operatorname{Tr}\left(T_{\mathbf{R}}^{\alpha} T_{\mathbf{R}}^{i} T_{\mathbf{R}}^{j} T_{\mathbf{R}}^{k}\right) & =d^{\alpha i j k} I_{4}\left(\mathbf{R}_{M}\right) \\
& =\frac{1}{4} \sum_{\mathbf{R}_{N} \subset \mathbf{R}_{M}} q_{\alpha}\left(\mathbf{R}_{N}\right) I_{3}\left(\mathbf{R}_{N}\right) d^{i j k} .
\end{aligned}
$$

This shows how the $\alpha_{i}$ and $\beta_{i}$ coefficients of $U(1) \otimes$ $S U(N)$ arise from the $\gamma_{4, i}$ coefficients of the general $S U(M \geq N+1)$ effective Lagrangian. Computationally, to check these identities requires first to work out the relationship between the symmetric symbols. In general, all we can say from the block-diagonal structure of the generators is that $d_{M}^{\alpha \alpha i j}=\eta_{1} \delta^{i j}$ and $d_{M}^{\alpha i j k}=\eta_{2} d_{N}^{i j k}$ [see Eq. (B19)], but the proportionality constants $\eta_{1}$ and $\eta_{2}$ depend on how $U(1) \otimes S U(N)$ is embedded into $S U(M)$. This is illustrated in Appendix B, where Eq. (53) is used to derive the quartic Casimir invariant $I_{4}$ of $S U(5)$ out of the anomaly coefficients $I_{3}$ of $S U(3)$.

As an interesting corollary of this exact reduction, the identities in Eq. (38) remain valid and imply $\alpha_{1,2}=\alpha_{3,4} / 2$. So, there are only two independent operators at the one loop level, no matter the spin and representation of the particle in the loop. As before, this is not true in general if more than a single field is integrated out. For example, the analogue of the Higgs boson exchange shown in Fig. 5(b) contributes to $\alpha_{1}$ only since the effective Higgs boson couplings to photons and gluons are $h^{0} F_{\mu \nu} F^{\mu \nu}$ and $h^{0} G_{\mu \nu}^{a} G^{a, \mu \nu}$.

\section{CONCLUSION}

In this paper, the effective action for gauge theories is revisited. Integrating out some heavy charged fields, selfinteractions among gauge bosons are encoded into effective operators. Using the diagrammatic approach, we explicitly constructed these interactions up to the dimension-eight level, and computed their coefficients as induced by loops of heavy particles of spin $0,1 / 2$, or 1 . More specifically,

(i) To set the stage and identify possible issues, we first reviewed in details the construction of the off shell effective couplings for photons. In the diagrammatic approach, integrating out fermions or scalars is straightforward and we recover the usual EulerHeisenberg result. For heavy vector fields, the matching does not proceeds as trivially. Indeed, in the 't Hooft-Feynman gauge, the gauge-fixing term required for the massive vector fields breaks the $U(1)$ gauge invariance. Consequently, the off shell four-photon amplitude fails to satisfy the QED Ward identities, and the usual procedure to construct the effective action breaks down. To solve this problem, we adopted the strategy of Ref. [33] and quantized the SM in the nonlinear gauge. Matching is then consistent off shell, and the diagrammatic approach closely parallels the path integral-based Covariant Derivative Expansion method [26,27]. The Wilson coefficients in that gauge are shown in Table I.

(ii) The calculation of the photon EFT was then extended to the QCD gluon EFT. The most general basis of gluonic operators up to dimension-eight is quite different from the QED case due to the non-Abelian nature of QCD [25]. We computed explicitly the coefficients of the effective operators for a scalar, fermion or vector in the fundamental representation. The final results for the coefficients are given in Table II. As for photons, integrating out heavy vector fields requires dealing with gauge dependences. Our strategy was to use the minimal $S U(5)$ GUT model, spontaneously broken by an adjoint Higgs scalar down to the unbroken SM gauge group, and quantized using a nonlinear gauge condition preserving the SM gauge invariance. Twelve of the $S U(5)$ gauge bosons become massive in the process, and those fields have precisely the quantum numbers needed to induce the effective gluon couplings. This construction is detailed in Appendix A. Technically, it should be mentioned that this nonlinear gauge has the additional nice feature of drastically reducing the number of diagrams for a given process.

(iii) We then extended the computation done in the QCD case to generic Lie gauge groups, taking $S U(N)$, $U(1) \otimes S U(N)$, and $S U(M) \otimes S U(N)$ as examples, and allowing the heavy particle to sit in arbitrary representations. The coefficients for a complex field of spin $0,1 / 2$, or 1 circulating in the loops are given in Table III for $S U(N)$ and in Table IV for nonsimple gauge groups. One feature apparent in these tables is worth stressing. At one loop, some operators are redundant no matter the representation or spin of the particle circulating in the loops. From our Eq. (38), we conclude that two operator combinations never 
occur in the one-loop effective action for $S U(N)$ gauge bosons. This implies in particular that only four instead of six operators are required for QCD, and only two instead of four operators are sufficient to describe the two gluon-two photon interactions. Finally, it should be mentioned that generalizing the QCD result to an arbitrary Lie algebra required a careful analysis of quartic Casimir invariants. While all the needed information can be dig out of the available literature [34,35], it seems to us a short review detailing all the definitions and conventions, and with emphasis on practical use in loop calculations, was lacking and so, it is included in Appendix B.

(iv) On a more technical note, the relationship between effective action and Feynman diagram matching was carefully analyzed. Specifically, the effective action can be computed from the one-loop 1PI off shell amplitudes. In this way, the coefficients of all the operators, including those vanishing under the equation of motion, are obtained. However, these coefficients are not necessarily gauge-invariant. Actually, since the matching is possible only using a nonlinear gauge fixing, they are well-defined in that gauge only. This is to be compared to the computation of the coefficients using on shell processes, where the physical on shell one-loop amplitudes are matched onto a subset of operators. Those operators that vanish under the EOM are absent, so the whole effective action is never reproduced. Further, from a calculation point of view, matching with on shell processes requires dealing with both 1PI and non-1PI amplitudes. For example, the coefficient of the three-gluon-field strength operator $f^{a b c} G_{\mu}^{a \nu} G_{\nu}^{b \rho} G_{\rho}^{c \mu}$ cannot be obtained from a three-gluon process since it is kinematically forbidden. Instead, it has to be extracted alongside all the four-gluon-field strength operators by matching onto the four-gluon physical amplitudes.

Altogether, the construction of the effective gauge-boson Lagrangian up to dimension-eight is now fully under control in the diagrammatic approach. The operator bases are confirmed, their group-theoretic properties clarified, and the coefficients are known for the standard benchmark scenarios of heavy scalars, fermions, and vector bosons.
Phenomenologically, though the four-gluon or four weak boson effective couplings is unlikely to be ever seen, given the presence of such a coupling in the tree-level Lagrangian, there may be some room for $\gamma \gamma \rightarrow g g$. In any case, having laid out a well-defined strategy to construct fully general effective actions involving gauge bosons will prove useful in the future.

\section{APPENDIX A: $S U(5)$ GAUGE BOSONS IN THE NONLINEAR GAUGE}

This Appendix is not intended as a review of the minimal $S U(5)$ model. Rather, it is meant as a guide to construct the Lagrangian of $S U(5)$ broken down to $S U(3)_{C} \otimes S U(2)_{L} \otimes$ $U(1)_{Y}$, quantized using a nonlinear gauge-fixing term, in a form suitable for automatic calculation tools. The main point is to input all the Lagrangian terms in a consistent and tractable way. This requires to set a number of conventions and definitions, so we found it useful to detail them here.

The starting point is to input the $S U(5)$ gauge bosons, and write them in terms of those of the $S U(3)_{C} \otimes$ $S U(2)_{L} \otimes U(1)_{Y}$ gauge group. For that, we start from the branching rule of the adjoint representation 24 :

$\mathbf{2 4}=(\mathbf{8}, \mathbf{1})_{0}+(\mathbf{1}, \mathbf{3})_{0}+(\mathbf{3}, \mathbf{2})_{5}+(\overline{\mathbf{3}}, \mathbf{2})_{-5}+(\mathbf{1}, \mathbf{1})_{0}$.

Denoting by $A, B, \ldots=1, \ldots, 24$ the $S U(5)$ adjoint indices, $a, b, \ldots=1, \ldots, 8$ the adjoint color indices, and $i, j, \ldots=1,2,3$ the fundamental $S U(3)$ indices, the twenty-four $A_{A}^{\mu}$ gauge bosons are identified as the octet of gluons $(\mathbf{8} \otimes \mathbf{1})_{0} \sim G_{i}^{\mu}=A_{i}^{\mu}, a=1, \ldots, 8$, the triplet of weak bosons $(\mathbf{1} \otimes \mathbf{3})_{0} \sim W^{ \pm \mu}=\left(A_{9}^{\mu} \mp i A_{10}^{\mu}\right) / \sqrt{2}, W_{3}^{\mu}=A_{11}^{\mu}$, and the singlet $(\mathbf{1} \otimes \mathbf{1})_{0} \sim B^{\mu}=A_{24}^{\mu}$. The remaining fields are the twelve leptoquark gauge bosons and their conjugate fields in the $(\overline{\mathbf{3}} \otimes \mathbf{2})_{5 / 3}$ and $(\mathbf{3} \otimes \overline{\mathbf{2}})_{-5 / 3}$ representation, respectively. We define these fields as $X_{1}^{\mu \pm}=\left(A_{12}^{\mu} \pm i A_{13}^{\mu}\right) /$ $\sqrt{2}, Y_{1}^{\mu \pm}=\left(A_{18}^{\mu} \pm i A_{19}^{\mu}\right) / \sqrt{2}$ and so on. Note that leptoquarks are charged under all the SM gauge groups, and those with positive hypercharge transform like antiquarks under $S U(3)_{C}$.

Since the adjoint is contained in $\mathbf{5} \otimes \overline{\mathbf{5}}=\mathbf{2 4} \oplus \mathbf{1}$, all these identifications of the gauge fields can be put together to construct a traceless $5 \times 5$ matrix for the $S U(5)$ gauge fields:

$$
\mathbf{A}^{\mu}=A_{A}^{\mu} T^{A}=\left(\begin{array}{ccc}
T_{i j}^{a} G_{\mu}^{a}-\frac{1}{\sqrt{15}} B_{\mu} \delta_{i j} & \frac{1}{\sqrt{2}} X_{\mu}^{i-} & \frac{1}{\sqrt{2}} Y_{\mu}^{i-} \\
\frac{1}{\sqrt{2}} X_{\mu}^{j+} & \frac{1}{2} W_{\mu}^{3}+\frac{3}{2 \sqrt{15}} B_{\mu} & \frac{1}{\sqrt{2}} W_{\mu}^{+} \\
\frac{1}{\sqrt{2}} Y_{\mu}^{j+} & \frac{1}{\sqrt{2}} W_{\mu}^{-} & -\frac{1}{2} W_{\mu}^{3}+\frac{3}{2 \sqrt{15}} B_{\mu}
\end{array}\right)
$$

where $T^{A}$ are the conventional $S U(5)$ generators in the fundamental representation, normalized as $\operatorname{Tr}\left(T^{A} T^{B}\right)=\delta^{A B} / 2$. This identification is compatible with the eigenstates of the electric charge operator, 


$$
Q=T^{11}+\sqrt{5 / 3} T^{24}
$$

$\left[Q, \mathbf{A}_{\mu}\right]=\frac{1}{\sqrt{2}}\left(\begin{array}{ccc}0 & -4 / 3 X_{\mu}^{i-} & -1 / 3 Y_{\mu}^{i-} \\ 4 / 3 X_{\mu}^{j+} & 0 & +W_{\mu}^{+} \\ 1 / 3 Y_{\mu}^{j+} & -W_{\mu}^{-} & 0\end{array}\right)$,

with the normalization of the hypercharge operator $Y=$ $2 \sqrt{5 / 3} T^{24}$. In practice, we have used the Mathematica package FeynArts [45] and FeynCalc [46]. Both allow to keep the summations over the $S U(3)$ indices as implicit, so $\mathbf{A}^{\mu}$ is truly input as the $3 \times 3$ matrix of Eq. (A2). Once all the relevant pieces of the Lagrangian are encoded, it is then a simple matter to extract the Feynman rules and export them to FEYnARTs. Let us now review the Lagrangian terms of relevance to us.

\section{Gauge interactions}

The gauge self-couplings derive from the Yang-Mills kinetic term

$$
\mathcal{L}_{\text {gauge }}=-\frac{1}{2}\left\langle\mathbf{A}_{\mu \nu} \mathbf{A}^{\mu \nu}\right\rangle=-\frac{1}{4} A_{\mu \nu}^{A} A^{A, \mu \nu},
$$

with the field strength

$$
\begin{aligned}
\mathbf{A}_{\mu \nu} & =\partial_{\mu} \mathbf{A}_{\nu}-\partial_{\nu} \mathbf{A}_{\mu}-i g_{5}\left[\mathbf{A}_{\mu}, \mathbf{A}_{\nu}\right] \\
& =\left(\partial_{\mu} A_{\nu}^{A}-\partial_{\nu} A_{\mu}^{A}+g f^{A B C} A_{\mu}^{B} A_{\nu}^{C}\right) T^{A} .
\end{aligned}
$$

The $S U(5)$ structure constants are defined as $\left[T^{A}, T^{B}\right]=$ if ${ }^{A B C} T^{C}$. An explicit calculation shows that there are 68 nonzero $f^{A B C}$, plus antisymmetric permutations of the indices. Among them there are the nine $f^{a b c}$ of $S U(3)$ and the single $\varepsilon^{i j k}$ of $S U(2)$, which reproduce the QCD and electroweak self-interactions. All the other nonzero structure constants are $f^{A B C}$ with $A, B=12, \ldots, 23$ and $C=1, \ldots, 11,24$. In other words, they involve twice the leptoquark fields, as can be expected since these particles are charged under the three SM gauge groups. The same $g_{5}$ occurs for all the interactions between gauge bosons. In explicit form,

$$
\begin{aligned}
\mathcal{L}_{\text {gauge }}= & -\frac{1}{2}\left\langle\left(\partial_{\mu} \mathbf{A}_{\nu}-\partial_{\nu} \mathbf{A}_{\mu}\right)\left(\partial^{\mu} \mathbf{A}^{\nu}-\partial^{\nu} \mathbf{A}^{\mu}\right)+4 i g_{5} \mathbf{A}_{\mu} \mathbf{A}_{\nu}\left(\partial^{\mu} \mathbf{A}^{\nu}-\partial^{\nu} \mathbf{A}^{\mu}\right)-2 g_{5}^{2} \mathbf{A}_{\mu} \mathbf{A}_{\nu}\left[\mathbf{A}^{\mu}, \mathbf{A}^{\nu}\right]\right\rangle \\
= & -\frac{1}{4} G_{\mu \nu}^{a} G^{a, \mu \nu}-\frac{1}{2} W_{\mu \nu}^{+} W^{-, \mu \nu}-\frac{1}{4} W_{\mu \nu}^{3} W^{3, \mu \nu}-\frac{1}{4} B_{\mu \nu} B^{\mu \nu} \\
& -\frac{1}{2}\left(D_{\mu} X_{\nu}^{+}-D_{\nu} X_{\mu}^{+}\right)^{i}\left(D^{\mu} X^{-\nu}-D^{\nu} X^{-\mu}\right)^{i}-\frac{1}{2}\left(D_{\mu} Y_{\nu}^{+}-D_{\nu} Y_{\mu}^{+}\right)^{i}\left(D^{\mu} Y^{-\nu}-D^{\nu} Y^{-\mu}\right)^{i} \\
& +i g_{5} G_{\mu \nu}^{a}\left(X_{\mu}^{j+}\left(-T_{j i}^{a}\right) X_{\nu}^{i-}+Y_{\mu}^{j+}\left(-T_{j i}^{a}\right) Y_{\nu}^{i-}\right)+i \frac{g_{5}}{\sqrt{2}}\left(W^{+, \mu \nu} Y_{\mu}^{i+} X_{\nu}^{i-}+W^{-, \mu \nu} X_{\mu}^{i+} Y_{\nu}^{i-}\right) \\
& +i \frac{g_{5}}{2} W^{3, \mu \nu}\left(X_{\mu}^{i+} X_{\nu}^{i-}-Y_{\mu}^{i+} Y_{\nu}^{i-}\right)+i g_{5} \frac{\sqrt{15}}{6} B^{\mu \nu}\left(X_{\mu}^{i+} X_{\nu}^{i-}+Y_{\mu}^{i+} Y_{\nu}^{i-}\right)+\mathcal{O}\left((X, Y)^{4}\right),
\end{aligned}
$$

where the weak and strong field strengths are understood to contain their respective non-Abelian terms, as

$$
\begin{aligned}
G_{\mu \nu}^{a}=\partial_{\nu} G_{\mu}^{a}-\partial_{\mu} G_{\nu}^{a}+g_{5} f^{a b c} G_{\mu}^{b} G_{\nu}^{c} & \rightarrow G_{\mu \nu}^{a} T^{a}=\partial_{\nu} G_{\mu}^{a} T^{a}-\partial_{\mu} G_{\nu}^{a} T^{a}-i g_{5}\left[G_{\mu}^{b} T^{b}, G_{\nu}^{c} T^{c}\right], \\
W^{i, \mu \nu}=\partial_{\nu} W_{\mu}^{i}-\partial_{\mu} W_{\nu}^{i}+g_{5} \varepsilon^{i j k} W_{\mu}^{j} W_{\nu}^{k} & \rightarrow\left\{\begin{array}{l}
W^{3, \mu \nu}=\partial_{\nu} W_{\mu}^{3}-\partial_{\mu} W_{\nu}^{3}+i g_{5}\left(W_{\mu}^{-} W_{\nu}^{+}-W_{\mu}^{+} W_{\nu}^{-}\right), \\
W^{+, \mu \nu}=\partial_{\nu} W_{\mu}^{+}-\partial_{\mu} W_{\nu}^{+}+i g_{5}\left(W_{\mu}^{+} W_{\nu}^{3}-W_{\mu}^{3} W_{\nu}^{+}\right), \\
W^{-, \mu \nu}=\partial_{\nu} W_{\mu}^{-}-\partial_{\mu} W_{\nu}^{-}+i g_{5}\left(W_{\mu}^{3} W_{\nu}^{-}-W_{\mu}^{-} W_{\nu}^{3}\right) .
\end{array}\right.
\end{aligned}
$$

The covariant derivative $D^{\mu}=\partial^{\mu} \mathbf{1}-i g_{5} T^{A} A_{A}^{\mu}$ acting on the twelve leptoquarks living in the $(\overline{\mathbf{3}} \otimes \mathbf{2})_{5 / 3}$ representation is

$$
\begin{aligned}
& \left(D_{\mu}\right)_{i j} X_{\nu}^{j+}=\partial_{\mu} X_{\nu}^{i+}-i g_{5}\left(X_{\nu}^{j+}\left(-T_{j i}^{a}\right) G_{\mu}^{a}+\frac{1}{2} W_{\mu}^{3} X_{\nu}^{i+}+\frac{1}{\sqrt{2}} W_{\mu}^{+} Y_{\nu}^{i+}+y \frac{5}{6} B_{\mu} X_{\nu}^{i+}\right) \\
& \left(D_{\mu}\right)_{i j} Y_{\nu}^{j+}=\partial_{\mu} Y_{\nu}^{i \pm}-i g_{5}\left(Y_{\nu}^{j+}\left(-T_{j i}^{a}\right) G_{\mu}^{a}-\frac{1}{2} W_{\mu}^{3} Y_{\nu}^{i+}+\frac{1}{\sqrt{2}} W_{\mu}^{-} X_{\nu}^{i+}+y \frac{5}{6} B_{\mu} Y_{\nu}^{i+}\right)
\end{aligned}
$$

where $y=\sqrt{3 / 5}$ is the hypercharge normalization. Finally, $\mathcal{O}\left((X, Y)^{4}\right)$ denotes quartic interactions among $X$ and $Y$ gauge bosons which are of no interest for our purpose. It is interesting to remark that the SM gauge invariance is satisfied separately for the $X, Y$ kinetic terms (thanks to the covariant derivatives), the magnetic interactions (the $B_{\mu \nu} X^{\mu} X^{\nu}$ and similar), and the $\mathcal{O}\left((X, Y)^{4}\right)$ interactions. At the level of the SM, the strength of the magnetic and $\mathcal{O}\left((X, Y)^{4}\right.$ interactions are thus unconstrained, and these could even be absent. On the contrary, 
here their relative strengths is fixed by the underlying $S U(5)$ gauge invariance. The situation is similar in the $\mathrm{SM}$, with the relative strength of the $\left(D_{\mu} W_{\nu}^{+}-D_{\nu} W_{\mu}^{+}\right)$ $\left(D^{\mu} W^{-\nu}-D^{\nu} W^{-\mu}\right)$ and $F_{\mu \nu} W_{\mu}^{+} W^{-\nu}$ interactions fixed by the underlying $S U(2)_{L} \otimes U(1)_{Y}$ symmetry.

\section{Scalar interactions}

In the present work, we are only interested in the initial breaking stage

$$
S U(5) \rightarrow S U(3)_{C} \otimes S U(2)_{L} \otimes U(1)_{Y} .
$$

For that, we need a scalar in the adjoint representation, $\overline{\mathbf{H}}_{\mathbf{2 4}}=\sqrt{2} H_{A} T_{A}$. Note that $\overline{\mathbf{H}}_{\mathbf{2 4}}=\overline{\mathbf{H}}_{\mathbf{2 4}}^{\dagger}$, since the adjoint is a real representation, and further assuming a $\overline{\mathbf{H}}_{\mathbf{2 4}} \rightarrow-\overline{\mathbf{H}}_{\mathbf{2 4}}$ symmetry to get rid of cubic interactions, the most general Lagrangian is

$\mathcal{L}_{\text {scalar }}=\frac{1}{2}\left\langle D_{\mu} \overline{\mathbf{H}}_{\mathbf{2 4}} D^{\mu} \overline{\mathbf{H}}_{\mathbf{2 4}}\right\rangle+\frac{\mu^{2}}{2}\left\langle\mathbf{H}_{\mathbf{2 4}}^{2}\right\rangle-\frac{a}{4}\left\langle\mathbf{H}_{\mathbf{2 4}}^{2}\right\rangle^{2}-\frac{b}{2}\left\langle\mathbf{H}_{\mathbf{2 4}}^{4}\right\rangle$.
The breaking of the $S U(5)$ symmetry arises when $\overline{\mathbf{H}}_{24}$ gets its vacuum expectation value $\left\langle 0\left|\overline{\mathbf{H}}_{\mathbf{2 4}}\right| 0\right\rangle \sim v_{5}>0$, which happens for $\mu^{2}>0$. There are two classes of minima, depending on the sign of $b$. First, it is possible to find values of $\mu, a$, and $b<0$ such that the minimum is of the form $\left\langle 0\left|\overline{\mathbf{H}}_{\mathbf{2 4}}\right| 0\right\rangle=\operatorname{diag}(v, v, v, v,-4 v)$. This corresponds to $S U(5) \rightarrow S U(4) \otimes U(1)$. The second class occurs for $b>0$ and is such that $\left\langle 0\left|\overline{\mathbf{H}}_{\mathbf{2 4}}\right| 0\right\rangle$ commutes with the $S U(3)_{C}, S U(2)_{L}$, and $U(1)_{Y}$ generators:

$$
\begin{aligned}
\mathbf{H}_{\mathbf{2 4}}^{0}=\left\langle 0\left|\overline{\mathbf{H}}_{\mathbf{2 4}}\right| 0\right\rangle & =\frac{1}{\sqrt{2}} v_{5} \operatorname{diag}(1,1,1,-3 / 2,-3 / 2) \\
& =-v_{5} \sqrt{15 / 4} T^{24}, \quad v_{5}^{2}=\frac{4 \mu^{2}}{15 a+7 b} .
\end{aligned}
$$

The value of $v_{5}$ is found by requiring that this is a global minimum of the potential, which asks for $15 a+7 b>0$.

Plugging this constraint in the scalar potential and writing

$$
\mathbf{H}_{\mathbf{2 4}}=\overline{\mathbf{H}}_{\mathbf{2 4}}-\mathbf{H}_{\mathbf{2 4}}^{0}=\sqrt{2}\left(\begin{array}{ccc}
T_{i j}^{a} H_{G}^{a}-\frac{1}{\sqrt{15}} H_{B}^{0} \delta_{i j} & \frac{1}{\sqrt{2}} H_{X}^{i-} & \frac{1}{\sqrt{2}} H_{Y}^{i-} \\
\frac{1}{\sqrt{2}} H_{X}^{j+} & \frac{1}{2} H_{W}^{3}+\frac{3}{2 \sqrt{15}} H_{B}^{0} & \frac{1}{\sqrt{2}} H_{W}^{+} \\
\frac{1}{\sqrt{2}} H_{Y}^{j+} & \frac{1}{\sqrt{2}} H_{W}^{-} & -\frac{1}{2} H_{W}^{3}+\frac{3}{2 \sqrt{15}} H_{B}^{0}
\end{array}\right),
$$

$$
\begin{aligned}
\mathcal{L}_{\text {mass }} & =-\frac{1}{2} g_{5}^{2}\left\langle\left[\mathbf{A}_{\mu}, \mathbf{H}_{\mathbf{2 4}}^{0}\right]\left[\mathbf{A}^{\mu}, \mathbf{H}_{\mathbf{2 4}}^{0}\right]\right\rangle \\
& =\frac{25}{16} g_{5}^{2} v_{5}^{2}\left(X_{\mu}^{i+} X^{i-\mu}+Y_{\mu}^{i+} Y^{i-\mu}\right),
\end{aligned}
$$

so $M_{X Y}=5 g_{5} v_{5} / 4$. The $\mathcal{L}_{\text {mix }}$ piece induces mixings between the $X^{\mu}$ and $Y^{\mu}$ gauge bosons and their associated WBG bosons,

$$
\begin{aligned}
\mathcal{L}_{\text {mix }} & =-i g_{5}\left\langle\left[\mathbf{A}_{\mu}, \mathbf{H}_{\mathbf{2 4}}^{0}\right] \partial^{\mu} \mathbf{H}_{\mathbf{2 4}}\right\rangle \\
& =i M_{X Y} X_{\mu}^{k-} \partial^{\mu} H_{X}^{k+}+i M_{X Y} Y_{\mu}^{k-} \partial^{\mu} H_{Y}^{k+}+\text { H.c. }
\end{aligned}
$$

The other couplings involve gauge and scalar bosons,

$$
\begin{aligned}
\mathcal{L}_{\text {gauge-Higgs }}= & -i g_{5}\left\langle\left[\mathbf{A}_{\mu}, \mathbf{H}_{\mathbf{2 4}}\right] \partial^{\mu} \mathbf{H}_{\mathbf{2 4}}\right\rangle \\
& -g_{5}^{2}\left\langle\left[\mathbf{A}_{\mu}, \mathbf{H}_{\mathbf{2 4}}^{0}\right]\left[\mathbf{A}^{\mu}, \mathbf{H}_{\mathbf{2 4}}\right]\right\rangle \\
& -\frac{g_{5}^{2}}{2}\left\langle\left[\mathbf{A}_{\mu}, \mathbf{H}_{\mathbf{2 4}}\right]\left[\mathbf{A}^{\mu}, \mathbf{H}_{\mathbf{2 4}}\right]\right\rangle .
\end{aligned}
$$

The explicit forms can easily be worked out and will not be given here. Remark though that because all the SM gauge bosons disappear from $\left[\mathbf{A}_{\mu}, \mathbf{H}_{24}^{0}\right], \mathcal{L}_{\mathrm{AAH}}$ only couples scalars to the massive gauge bosons, with couplings proportional to their mass.

The $\mathcal{L}_{\text {mass }}$ couplings are just the leptoquark mass terms, 


\section{Gauge-fixing and ghost interactions}

The next step to quantize this theory is to fix the gauge, and add the corresponding ghost terms. The general ansatz in linear $R_{\xi}$ gauge is to define the constraint in terms of the WBG as

$$
\begin{aligned}
\mathbf{G} & =\sqrt{2} \partial_{\mu} \mathbf{A}^{\mu}+\xi M_{X Y}\left(\begin{array}{ccc}
0 & i H_{X}^{i-} & i H_{Y}^{i-} \\
-i H_{X}^{j+} & 0 & 0 \\
-i H_{Y}^{j+} & 0 & 0
\end{array}\right) \\
& =\left(\begin{array}{ccc}
\sqrt{2} T_{i j}^{a} \partial^{\mu} G_{\mu}^{a}-\sqrt{\frac{2}{15}} \partial^{\mu} B_{\mu} \delta_{i j} & \partial^{\mu} X_{\mu}^{i-}+i \xi M_{X Y} H_{X}^{i-} & \partial^{\mu} Y_{\mu}^{i-}+i \xi M_{X Y} H_{Y}^{i-} \\
\partial^{\mu} X_{\mu}^{j+}-i \xi M_{X Y} H_{X}^{j+} & \frac{1}{\sqrt{2}} \partial^{\mu} W_{\mu}^{3}+\sqrt{\frac{3}{10}} \partial^{\mu} B_{\mu} & \partial^{\mu} W_{\mu}^{+} \\
\partial^{\mu} Y_{\mu}^{j+}-i \xi M_{X Y} H_{Y}^{j+} & \partial^{\mu} W_{\mu}^{-} & -\frac{1}{\sqrt{2}} \partial^{\mu} W_{\mu}^{3}+\frac{3}{2 \sqrt{15}} \partial^{\mu} B_{\mu}
\end{array}\right),
\end{aligned}
$$

so that

$$
\begin{aligned}
\mathcal{L}_{\mathrm{gf}}=- & \frac{1}{2 \xi}\left\langle\mathbf{G}^{2}\right\rangle=-\frac{1}{\xi}\left|\partial^{\mu} X_{\mu}^{k+}-i \xi M_{X Y} H_{X}^{k+}\right|^{2}-\frac{1}{\xi}\left|\partial^{\mu} Y_{\mu}^{k+}-i \xi M_{X Y} H_{Y}^{k+}\right|^{2} \\
& -\frac{1}{\xi}\left|\partial^{\mu} W_{\mu}^{+}\right|^{2}-\frac{1}{2 \xi}\left(\partial^{\mu} W_{\mu}^{3}\right)^{2}-\frac{1}{2 \xi}\left(\partial^{\mu} B_{\mu}\right)^{2}-\frac{1}{2 \xi}\left(\partial^{\mu} G_{\mu}^{a}\right)^{2}
\end{aligned}
$$

Since in practice, all our computations are done in the 't Hooft-Feynman gauge, a common parameter $\xi$ is introduced for all the gauge bosons. Obviously, the parameters for $G_{\mu}^{a}, B_{\mu}, W_{\mu}^{3}$, and $W_{\mu}^{ \pm}$can be all different since they appear only in the respective propagator and not in any of the vertices. For $X_{\mu}^{i \pm}$ and $Y_{\mu}^{i \pm}$, not taking a common parameter would make life more complicated since those two form an $S U(2)_{L}$ doublet. When the first line is expanded, the terms linear in $M_{X Y}$ precisely cancel those in $\mathcal{L}_{\text {mix }}$, while those quardratic imply $M_{H_{X Y}}^{2}=\xi M_{X Y}^{2}$ as usual. Remember that WBG do not get any mass term from the scalar potential.

The goal of the nonlinear gauge fixing of Ref. [57] is to maintain the unbroken gauge symmetries as explicit. This requires general covariant derivatives in the constraints involving the massive gauge bosons. To be able to interpolating between the linear and nonlinear gauge, we introduce the parameters $\alpha_{G}, \alpha_{W}, \alpha_{B}$ and use

$$
\begin{aligned}
\partial^{\mu} X_{\mu}^{i+} \rightarrow & \partial^{\mu} X_{\mu}^{i+}-i g_{5}\left(\alpha_{G} X_{\nu}^{j+}\left(-T_{j i}^{a}\right) G_{\mu}^{a}+\alpha_{W} \frac{1}{2} W_{\mu}^{3} X_{\nu}^{i+}\right. \\
& \left.+\alpha_{W} \frac{1}{\sqrt{2}} W_{\mu}^{+} Y_{\nu}^{i+}+\alpha_{B} y \frac{5}{6} B_{\mu} X_{\nu}^{i+}\right), \\
\partial^{\mu} Y_{\nu}^{i+} \rightarrow & \partial^{\mu} Y_{\mu}^{i \pm}-i g_{5}\left(\alpha_{G} Y_{\nu}^{j+}\left(-T_{j i}^{a}\right) G_{\mu}^{a}-\alpha_{W} \frac{1}{2} W_{\mu}^{3} Y_{\nu}^{i+}\right. \\
& \left.+\alpha_{W} \frac{1}{\sqrt{2}} W_{\mu}^{-} X_{\nu}^{i+}+\alpha_{B} y \frac{5}{6} B_{\mu} Y_{\nu}^{i+}\right) .
\end{aligned}
$$

Plugging this in $\mathcal{L}_{\text {gf }}$ generates new contributions to $\mathcal{L}_{\text {gauge }}$ and $\mathcal{L}_{\text {gauge-Higgs. }}$ At this stage, one of the interest of this gauge becomes apparent. The gauge and gauge-WBG Lagrangian of the previous section must be invariant under the SM gauge symmetry. This means that among the WBGgauge-gauge interactions, there are precisely those needed to promote the derivatives in $\mathcal{L}_{\text {mix }}$ to covariant ones. But then, having covariant derivatives in $\mathcal{L}_{\text {gf }}$ cancels them out. As a result, when $\alpha_{i}=1$, the $A-A-W B G$ couplings get much simpler.

To this constraint corresponds the ghost Lagrangian

$$
\mathcal{L}_{\text {ghost }}=c^{A \dagger}\left(\left.\left(-g_{5}\right) \frac{\delta G^{A}}{\delta \lambda^{B}}\right|_{\lambda=0}\right) c^{B} .
$$

To get the variation of $G^{A}$ under a gauge transformation, we first need that of the fields, expressed in the same physical basis as the gauge bosons and WBG scalars. For the gauge fields, the variation under a gauge transformation is

$$
\delta \mathbf{A}^{\mu}=\frac{1}{g_{5}} D_{\mu} \lambda=\frac{1}{g_{5}} \partial^{\mu} \lambda-i\left[\mathbf{A}^{\mu}, \boldsymbol{\lambda}\right]
$$

where the physical basis parameters are defined from $\lambda=$ $\lambda^{A} T^{A}$ in full analogy to the gauge bosons. In explicit form, reconstructing the individual field transformation, 


$$
\begin{gathered}
\delta G_{\mu}^{a}=\frac{1}{g_{5}} \partial^{\mu} \lambda_{G}^{a}+f^{a b c} G_{\mu}^{b} \lambda_{G}^{c}+i\left(X_{\mu}^{i+} T_{i j}^{a} \lambda_{X}^{j-}-\lambda_{X}^{i+} T_{i j}^{a} X_{\mu}^{j-}+Y_{\mu}^{i+} T_{i j}^{a} \lambda_{Y}^{j-}-\lambda_{Y}^{i+} T_{i j}^{a} Y_{\mu}^{j-}\right), \\
\delta W_{\mu}^{+}=\frac{1}{g_{5}} \partial^{\mu} \lambda_{W}^{+}+i W_{\mu}^{+} \lambda_{W}^{3}-i W_{\mu}^{3} \lambda_{W}^{+}+\frac{i}{\sqrt{2}}\left(\lambda_{X}^{i+} Y_{\mu}^{i-}-\lambda_{Y}^{i-} X_{\mu}^{i+}\right), \quad \delta W_{\mu}^{-}=\left(\delta W_{\mu}^{+}\right)^{\dagger}, \\
\delta W_{\mu}^{3}=\frac{1}{g_{5}} \partial^{\mu} \lambda_{W}^{3}+i W_{\mu}^{-} \lambda_{W}^{+}-i W_{\mu}^{+} \lambda_{W}^{-}+\frac{i}{2}\left(\lambda_{X}^{i+} X_{\mu}^{i-}-\lambda_{X}^{i-} X_{\mu}^{i+}-\lambda_{Y}^{i+} Y_{\mu}^{i-}+\lambda_{Y}^{i-} Y_{\mu}^{i+}\right), \\
\delta B_{\mu}=\frac{1}{g_{5}} \partial^{\mu} \lambda_{B}+\frac{i}{2} \sqrt{\frac{5}{3}}\left(\lambda_{X}^{i+} X_{\mu}^{i-}-\lambda_{X}^{i-} X_{\mu}^{i+}+\lambda_{Y}^{i+} Y_{\mu}^{i-}-\lambda_{Y}^{i-} Y_{\mu}^{i+}\right), \\
\delta X_{\mu}^{i+}=\frac{1}{g_{5}} \partial^{\mu} \lambda_{X}^{i+}-i\left(\lambda_{X}^{k+} T_{k i}^{a} G_{\mu}^{a}-X_{\mu}^{k+} T_{k i}^{a} \lambda_{G}^{a}\right)+\frac{i}{\sqrt{2}}\left(\lambda_{W}^{+} Y_{\mu}^{i+}-\lambda_{Y}^{i+} W_{\mu}^{+}\right)+\frac{i}{2}\left(\lambda_{W}^{3} X_{\mu}^{i+}-\lambda_{X}^{i+} W_{\mu}^{3}\right)+\frac{i}{2} \sqrt{\frac{5}{3}}\left(\lambda_{B} X_{\mu}^{i+}-\lambda_{X}^{i+} B_{\mu}\right), \\
\delta X_{\mu}^{i-}=\left(\delta X_{\mu}^{i+}\right)^{\dagger}, \quad\left(\mathrm{A} 2{ }^{i+}\right. \\
\delta Y_{\mu}^{i+}=\frac{1}{g_{5}} \partial^{\mu} \lambda_{Y}^{i+}+i\left(\lambda_{Y}^{k+} T_{k i}^{a} G_{\mu}^{a}-Y_{\mu}^{k+} T_{k i}^{a} \lambda_{G}^{a}\right)+\frac{i}{\sqrt{2}}\left(\lambda_{W}^{-} X_{\mu}^{i+}-\lambda_{X}^{i+} W_{\mu}^{-}\right)-\frac{i}{2}\left(\lambda_{W}^{3} Y_{\mu}^{i+}-\lambda_{Y}^{i+} W_{\mu}^{3}\right)+\frac{i}{2} \sqrt{\frac{5}{3}}\left(\lambda_{B} Y_{\mu}^{i+}-\lambda_{Y}^{i+} B_{\mu}\right), \\
\delta Y_{\mu}^{i-}=\left(\delta Y_{\mu}^{i+}\right)^{\dagger} .
\end{gathered}
$$

Similarly, the transformation of the scalar fields in the adjoint representation $\delta H^{A}=f^{A B C} H^{B} \lambda^{C}$ can be obtained in matrix form

$$
g \delta \overline{\mathbf{H}}_{24}=i\left[\lambda, \overline{\mathbf{H}}_{24}\right] \rightarrow \delta \mathbf{H}_{24}=i\left[\lambda, \mathbf{H}_{24}\right]+i\left[\lambda, \mathbf{H}_{24}^{0}\right]
$$

We only need the transformation rule of the WBG, since the other scalar fields will not be introduced in the gauge constraints:

$$
\begin{gathered}
\delta H_{X}^{i+}=-i H_{X}^{k+} T_{k i}^{a} \lambda_{G}^{a}+\frac{i}{\sqrt{2}} \lambda_{W}^{+} H_{Y}^{i+}+\frac{i}{2} \lambda_{W}^{3} H_{X}^{i+}+\frac{i}{2} \sqrt{\frac{5}{3}} \lambda_{B} H_{X}^{i+}+i \frac{5}{4} v_{5} \lambda_{X}^{i+} \\
+i \lambda_{X}^{k+} T_{k i}^{a} H_{G}^{a}-\frac{i}{\sqrt{2}} H_{W}^{+} \lambda_{Y}^{i+}-\frac{i}{2} H_{W}^{3} \lambda_{X}^{i+}-\frac{i}{2} \sqrt{\frac{5}{3}} H_{B} \lambda_{X}^{i+}, \\
\delta H_{Y}^{i+}=-i H_{Y}^{k+} T_{k i}^{a} \lambda_{G}^{a}+\frac{i}{\sqrt{2}} \lambda_{W}^{-} H_{X}^{i+}-\frac{i}{2} \lambda_{W}^{3} H_{Y}^{i+}+\frac{i}{2} \sqrt{\frac{5}{3}} \lambda_{B} H_{Y}^{i+}+i \frac{5}{4} v_{5} \lambda_{Y}^{i+}+i \lambda_{Y}^{k+} T_{k i}^{a} H_{G}^{a} \\
-\frac{i}{\sqrt{2}} H_{W}^{-} \lambda_{X}^{i+}+\frac{i}{2} H_{W}^{3} \lambda_{Y}^{i+}-\frac{i}{2} \sqrt{\frac{5}{3}} H_{B} \lambda_{Y}^{i+} .
\end{gathered}
$$

Note that these transformation rules imply that only the ghost fields associated to the massive gauge bosons couple to all the Higgs bosons, as expected from the absence of direct couplings of the scalar fields to SM gauge bosons.

Once $\mathbf{G}$ is expressed in the physical basis [as in Eq. (A18) for the linear gauge], the physical gauge parameters identified from $\lambda$, and ghost matrices defined in full analogy as

$$
\mathbf{c}=c_{A} T^{A}=\left(\begin{array}{ccc}
T_{i j}^{a} c_{G}^{a}-\frac{1}{\sqrt{15}} c_{B} \delta_{i j} & \frac{1}{\sqrt{2}} c_{X}^{i-} & \frac{1}{\sqrt{2}} c_{Y}^{i-} \\
\frac{1}{\sqrt{2}} c_{X}^{j+} & \frac{1}{2} c_{W}^{3}+\frac{3}{2 \sqrt{15}} c_{B} & \frac{1}{\sqrt{2}} c_{W}^{+} \\
\frac{1}{\sqrt{2}} c_{Y}^{j+} & \frac{1}{\sqrt{2}} c_{W}^{-} & -\frac{1}{2} c_{W}^{3}+\frac{3}{2 \sqrt{15}} c_{B}
\end{array}\right)
$$

one can proceed by computing $-\sqrt{2} g_{5}\left\langle\mathbf{c}^{\dagger} \delta \mathbf{G}\right\rangle$ and replacing each $\lambda$ by the corresponding ghost, i.e., $\lambda_{B} \rightarrow c_{B}, \lambda_{G}^{a} \rightarrow c_{G}^{a}$, etc. Given the many possible couplings once a nonlinear gauge fixing is imposed, the final expression are very lengthy and will not be written down here. Let us just remark that only the ghosts associated to the leptoquarks get massive, 


$$
\begin{aligned}
\mathcal{L}_{\text {ghost }}= & c_{G}^{a^{\dagger}}\left(-\partial^{2}\right) c_{G}+c_{B}^{\dagger}\left(-\partial^{2}\right) c_{B}+c_{W}^{3 \dagger}\left(-\partial^{2}\right) c_{W}^{3}+c_{W}^{\dagger+}\left(-\partial^{2}\right) c_{W}^{-}+c_{W}^{\dagger-}\left(-\partial^{2}\right) c_{W}^{+} \\
& +c_{X}^{\dagger+}\left(-\partial^{2}-\xi M_{X Y}^{2}\right) c_{X}^{-}+c_{X}^{\dagger-}\left(-\partial^{2}-\xi M_{X Y}^{2}\right) c_{X}^{+}+c_{Y}^{\dagger+}\left(-\partial^{2}-\xi M_{X Y}^{2}\right) c_{Y}^{-}+c_{Y}^{\dagger-}\left(-\partial^{2}-\xi M_{X Y}^{2}\right) c_{Y}^{+} \\
& +\mathcal{L}_{\mathrm{CCV}}+\mathcal{L}_{\mathrm{CCH}}+\mathcal{L}_{\mathrm{CCVV}} .
\end{aligned}
$$

Still, the SM ghosts get new interactions with pairs of heavy states (one ghost, one gauge boson). Note also that $\mathcal{L}_{\mathrm{CCVV}}$ derives entirely from the nonlinear gauge fixing.

\section{APPENDIX B: CASIMIR INVARIANTS OF STANDARD LIE ALGEBRAS}

The structure constants of a simple Lie algebra are defined as $\left[T_{\mathbf{R}}^{a}, T_{\mathbf{R}}^{b}\right]=i f^{a b c} T_{\mathbf{R}}^{c}$, with $T_{\mathbf{R}}^{a}$ the generators in the representation $\mathbf{R}$. The quadratic and cubic Casimir invariants are defined in terms of the fully symmetrized trace over two and three generators

$$
\begin{aligned}
\frac{1}{2 !} S \operatorname{Tr}\left(T_{\mathbf{R}}^{a} T_{\mathbf{R}}^{b}\right) & =\operatorname{Tr}\left(T_{\mathbf{R}}^{a} T_{\mathbf{R}}^{b}\right) \equiv I_{2}(\mathbf{R}) d^{a b}, \\
\frac{1}{3 !} S \operatorname{Tr}\left(T_{\mathbf{R}}^{a} T_{\mathbf{R}}^{b} T_{\mathbf{R}}^{c}\right) & =\frac{1}{2} \operatorname{Tr}\left(T_{\mathbf{R}}^{a}\left\{T_{\mathbf{R}}^{b}, T_{\mathbf{R}}^{c}\right\}\right) \equiv \frac{1}{4} I_{3}(\mathbf{R}) d^{a b c} .
\end{aligned}
$$

In terms of these two invariants, we can reduce the trace over three generators as

$$
\begin{aligned}
\operatorname{Tr}\left(T_{\mathbf{R}}^{a} T_{\mathbf{R}}^{b} T_{\mathbf{R}}^{c}\right) & =\frac{1}{2} \operatorname{Tr}\left(\left[T_{\mathbf{R}}^{a}, T_{\mathbf{R}}^{b}\right] T_{\mathbf{R}}^{c}\right)+\frac{1}{2} \operatorname{Tr}\left(\left\{T_{\mathbf{R}}^{a}, T_{\mathbf{R}}^{b}\right\} T_{\mathbf{R}}^{c}\right) \\
& =\frac{I_{3}(\mathbf{R})}{4} d^{a b c}+\frac{i I_{2}(\mathbf{R})}{2} f^{a b c} .
\end{aligned}
$$

The quadratic invariant defines a metric in the generator space. $\operatorname{Tr}\left(T_{\mathbf{R}}^{a} T_{\mathbf{R}}^{b}\right)$ being positive definite, it is always possible to choose a basis for the generators so that $d^{a b}=\delta^{a b}$. By convention, the generators are further normalized so that $I_{2}(\mathbf{F}) \equiv c$, with $\mathbf{F}$ the defining representation of dimension $N(\mathbf{F})=N$ and the constant $c$ usually set to $1 / 2$ or 1 . Note also that once $d^{a b}=\delta^{a b}$, $T_{\mathbf{R}}^{a} T_{\mathbf{R}}^{a}$ becomes proportional to the identity, with $T_{\mathbf{R}}^{a} T_{\mathbf{R}}^{a}=$ $\left(N(\mathbf{A}) I_{2}(\mathbf{R}) / N(\mathbf{R})\right) \mathbf{1}_{N(\mathbf{R}) \times N(\mathbf{R})}$ where $N(\mathbf{R})$ denotes the dimension of the representation $\mathbf{R}$, while $\mathbf{A}$ stands for the adjoint representation.

The totally symmetric tensor $d^{a b c}$ is normalized such that $I_{3}(\mathbf{F}) \equiv 1$ for unitary groups. It is absent for orthogonal groups, except for $S O(6)$ isomorphic to $S U(4)$. When defined, the coefficient $I_{3}(\mathbf{R})$ is often called the anomaly coefficient of the representation $\mathbf{R}$.

\section{Quartic symmetric symbol}

To compute traces over four generators, we need to extend the basis to include the quartic symmetric symbol and its associated invariant (for more information, see Ref. [35]). It is not immediately given by the fully symmetric trace over four generators because the symmetrized product of two second-order symmetric symbols is an invariant symmetric tensor with four indices. Specifically, the most general decomposition is:

$$
\begin{aligned}
& \frac{1}{4 !} S \operatorname{Tr}\left(T_{\mathbf{R}}^{a} T_{\mathbf{R}}^{b} T_{\mathbf{R}}^{c} T_{\mathbf{R}}^{d}\right) \\
& \quad=I_{4}(\mathbf{R}) d^{a b c d}+\Lambda(\mathbf{R})\left(\delta^{a b} \delta^{c d}+\delta^{a c} \delta^{b d}+\delta^{a d} \delta^{b c}\right) .
\end{aligned}
$$

The constant $\Lambda(\mathbf{R})$ is a matter of convention, while $d^{a b c d}$ is normalized by fixing $I_{4}(\mathbf{F})=c$ for some chosen constant $c$. To fix $\Lambda(\mathbf{R})$, we choose to define the tensor $d^{a b c d}$ as orthogonal to the lower rank invariants, i.e., such that $d_{a b} d_{c d} d^{a b c d}=0$ :

$$
\begin{aligned}
I_{4}(\mathbf{R}) d_{a b} d_{c d} d^{a b c d} & =\frac{1}{4 !} \delta_{a b} \delta_{c d} S \operatorname{Tr}\left(T_{\mathbf{R}}^{a} T_{\mathbf{R}}^{b} T_{\mathbf{R}}^{c} T_{\mathbf{R}}^{d}\right)-\delta_{a b} \delta_{c d} \Lambda(\mathbf{R})\left(\delta^{a b} \delta^{c d}+\delta^{a c} \delta^{b d}+\delta^{a d} \delta^{b c}\right) \\
& =\operatorname{Tr}\left(T_{\mathbf{R}}^{a} T_{\mathbf{R}}^{a} T_{\mathbf{R}}^{b} T_{\mathbf{R}}^{b}\right)+\frac{1}{3} \operatorname{Tr}\left(T_{\mathbf{R}}^{a}\left[T_{\mathbf{R}}^{b}, T_{\mathbf{R}}^{a}\right] T_{\mathbf{R}}^{b}\right)-\Lambda(\mathbf{R})(2+N(\mathbf{A})) N(\mathbf{A}) \\
& =\left(\frac{N(\mathbf{A}) I_{2}(\mathbf{R})}{N(\mathbf{R})}-\frac{I_{2}(\mathbf{A})}{6}\right) I_{2}(\mathbf{R}) N(\mathbf{A})-\Lambda(\mathbf{R})(2+N(\mathbf{A})) N(\mathbf{A}),
\end{aligned}
$$

where we have used $f^{a b c} f^{d b c}=I_{2}(\mathbf{A}) \delta^{a d}, f^{a b c} f^{a b c}=I_{2}(\mathbf{A}) N(\mathbf{A})$. Hence, $d_{a b} d_{c d} d^{a b c d}$ vanishes provided

$$
\Lambda(\mathbf{R})=\left(\frac{N(\mathbf{A}) I_{2}(\mathbf{R})}{N(\mathbf{R})}-\frac{I_{2}(\mathbf{A})}{6}\right) \frac{I_{2}(\mathbf{R})}{2+N(\mathbf{A})} .
$$

This convention ensures $d^{a b c d}$ has no left-over part proportional to the quadratic symbol. This is particularly convenient because $I_{4}(\mathbf{R})$ then vanishes for all $\mathbf{R}$ of $S U(2)$ and $S U(3)$. Remember that a tensor $d^{a b c d}$ such that $d_{a b} d_{c d} d^{a b c d}=0$ does not exist for $S U(N \leq 3)$. For $N=2,3, \Lambda(\mathbf{F})=1 / 24$ and 


$$
S \operatorname{Tr}\left(T_{\mathbf{F}}^{a} T_{\mathbf{F}}^{b} T_{\mathbf{F}}^{c} T_{\mathbf{F}}^{d}\right) \stackrel{N=2,3}{=} \delta^{a b} \delta^{c d}+\delta^{a c} \delta^{b d}+\delta^{a d} \delta^{b c}
$$

This formula also provides a useful identity for the $S U(3)$ structure constant:

$$
\begin{aligned}
\frac{1}{4 !} S \operatorname{Tr}\left(T_{\mathbf{8}}^{a} T_{\mathbf{8}}^{b} T_{\mathbf{8}}^{c} T_{\mathbf{8}}^{b}\right) & =\frac{1}{4 !} \sum_{\operatorname{perm}(a, b, c, d)} f^{a x_{1} x_{2}} f^{b x_{2} x_{3}} f^{c x_{3} x_{4}} f^{d x_{4} x_{1}} \\
& =\frac{3}{4}\left(\delta^{a b} \delta^{c d}+\delta^{a c} \delta^{b d}+\delta^{a d} \delta^{b c}\right),
\end{aligned}
$$

since $\Lambda(8)=3 / 4$.

The formula Eq. (B4) is valid for all unitary and orthogonal algebras, except for $S O(8)$. Indeed, the $N$ dimensional Levi-Civita symbol is an invariant for $S O(N)$, and when $N$ is even, it is possible to construct out of it a symmetric symbol with $N / 2$ indices. To see this, remember that the adjoint $\mathbf{A}$ of $S O(N)$ is obtained as the antisymmetric tensor product of the defining $N$-dimensional representation $\mathbf{F}, \mathbf{A}=\mathbf{F} \otimes_{A} \mathbf{F}$. Thus, the $S O(N)$ generators can be labelled by antisymmetric combinations of two indices $i, j=1, \ldots, N$. If we denote $a=(i, j)$, with $a=1, \ldots, N(N-1) / 2$, then

$$
\Theta^{a_{1} \ldots a_{N / 2}}=\eta \varepsilon^{i_{1} \ldots i_{N}}
$$

with $\eta$ some constants, is a totally symmetric invariant tensor with $N / 2$ indices. This explains one aspect of the isomorphism $S O(6) \sim S U(4)$. None of the orthogonal algebras have a genuine $d^{a b c}$ symbol, but the extra invariant tensor $\Theta^{a b c}$ of $S O(6)$ corresponds to the $d^{a b c}$ symbol of $S U(4)$. For $S O(8), \Theta^{a b c d}$ is an additional quartic symbol, orthogonal to both tensor structures in Eq. (B4). Thus, the totally symmetric trace over four $S O(8)$ generators projects not just on two but three tensor structures.

\section{Fourth-order trace reductions}

Any trace over four generators can be reduced and expressed entirely in terms of the invariant tensors. For instance, for $S U(N)$ and $S O(N \neq 8)$, we can write

$$
\begin{aligned}
\frac{1}{4 !} S \operatorname{Tr}\left(T_{\mathbf{R}}^{a} T_{\mathbf{R}}^{b} T_{\mathbf{R}}^{c} T_{\mathbf{R}}^{d}\right)= & \frac{1}{6} \operatorname{Tr}\left(T_{\mathbf{R}}^{a} T_{\mathbf{R}}^{b} T_{\mathbf{R}}^{c} T_{\mathbf{R}}^{d}\right)+\frac{1}{6} \operatorname{Tr}\left(T_{\mathbf{R}}^{a} T_{\mathbf{R}}^{b} T_{\mathbf{R}}^{d} T_{\mathbf{R}}^{c}\right)+\frac{1}{6} \operatorname{Tr}\left(T_{\mathbf{R}}^{a} T_{\mathbf{R}}^{c} T_{\mathbf{R}}^{b} T_{\mathbf{R}}^{d}\right) \\
& +\frac{1}{6} \operatorname{Tr}\left(T_{\mathbf{R}}^{a} T_{\mathbf{R}}^{c} T_{\mathbf{R}}^{d} T_{\mathbf{R}}^{b}\right)+\frac{1}{6} \operatorname{Tr}\left(T_{\mathbf{R}}^{a} T_{\mathbf{R}}^{d} T_{\mathbf{R}}^{b} T_{\mathbf{R}}^{c}\right)+\frac{1}{6} \operatorname{Tr}\left(T_{\mathbf{R}}^{a} T_{\mathbf{R}}^{d} T_{\mathbf{R}}^{c} T_{\mathbf{R}}^{b}\right) \\
= & \operatorname{Tr}\left(T_{\mathbf{R}}^{a} T_{\mathbf{R}}^{b} T_{\mathbf{R}}^{c} T_{\mathbf{R}}^{d}\right)+\frac{2}{6} i f^{d c e} \operatorname{Tr}\left(T_{\mathbf{R}}^{a} T_{\mathbf{R}}^{b} T_{\mathbf{R}}^{e}\right)+\frac{3}{6} i f^{c b e} \operatorname{Tr}\left(T_{\mathbf{R}}^{a} T_{\mathbf{R}}^{e} T_{\mathbf{R}}^{d}\right)+\frac{2}{6} i f^{d b e} \operatorname{Tr}\left(T_{\mathbf{R}}^{a} T_{\mathbf{R}}^{c} T_{\mathbf{R}}^{e}\right) \\
& +\frac{1}{6} i f^{d b e} \operatorname{Tr}\left(T_{\mathbf{R}}^{a} T_{\mathbf{R}}^{e} T_{\mathbf{R}}^{c}\right)+\frac{1}{6} i f^{d c e} \operatorname{Tr}\left(T_{\mathbf{R}}^{a} T_{\mathbf{R}}^{e} T_{\mathbf{R}}^{b}\right) \\
= & \operatorname{Tr}\left(T_{\mathbf{R}}^{a} T_{\mathbf{R}}^{b} T_{\mathbf{R}}^{c} T_{\mathbf{R}}^{d}\right)+i \frac{I_{3}(\mathbf{R})}{8}\left(f^{d c e} d^{a b e}+f^{c b e} d^{a e d}+f^{d b e} d^{a c e}\right)+\frac{I_{2}(\mathbf{R})}{12} f^{a b e} f^{c d e} \\
& -\frac{I_{2}(\mathbf{R})}{4} f^{a d e} f^{b c e}+\frac{I_{2}(\mathbf{R})}{12} f^{a c e} f^{b d e} .
\end{aligned}
$$

Or, introducing the quartic invariant:

$$
\begin{aligned}
\operatorname{Tr}\left(T_{\mathbf{R}}^{a} T_{\mathbf{R}}^{b} T_{\mathbf{R}}^{c} T_{\mathbf{R}}^{d}\right)= & I_{4}(\mathbf{R}) d^{a b c d}-i \frac{I_{3}(\mathbf{R})}{8}\left(f^{d c e} d^{a b e}+f^{c b e} d^{a e d}+f^{d b e} d^{a c e}\right) \\
& -\frac{I_{2}(\mathbf{R})}{12}\left(f^{a b e} f^{c d e}-3 f^{a d e} f^{b c e}+f^{a c e} f^{b d e}\right)+\Lambda(\mathbf{R})\left(\delta^{a b} \delta^{c d}+\delta^{a c} \delta^{b d}+\delta^{a d} \delta^{b c}\right)
\end{aligned}
$$

As special cases, we can set $I_{3}(\mathbf{R})=0$ for $S O(N \neq 6), I_{4}(\mathbf{R})=0$ for $S U(3)$, and $I_{4}(\mathbf{R})=I_{3}(\mathbf{R})=0$ for $S U(2)$. Note that the last two terms can be brought to a simpler though less symmetric form using the Jacobi identities:

$$
\begin{aligned}
& f^{c d e} d^{a b e}+f^{a d e} d^{b c e}+f^{b d e} d^{a c e}=0, \\
& f^{a b e} f^{c d e}-f^{a c e} f^{b d e}+f^{a d e} f^{b c e}=0 .
\end{aligned}
$$

Other identities sometimes useful in the computation of triangle graphs are:

$$
f^{a d e} f^{b e f} f^{c f d}=+\frac{1}{2} I_{2}(\mathbf{A}) f^{a b c}
$$




$$
d^{a d e} f^{b e f} f^{c f d}=-\frac{1}{2} I_{2}(\mathbf{A}) d^{a b c} .
$$

The first identity derives from $\operatorname{Tr}\left(T_{\mathbf{A}}^{a} T_{\mathbf{A}}^{b} T_{\mathbf{A}}^{c}\right)=\operatorname{Tr}\left(T_{\mathbf{A}}^{a}\left[T_{\mathbf{A}}^{b}, T_{\mathbf{A}}^{c}\right]\right) / 2$ since $\left(T_{\mathbf{A}}^{a}\right)^{T}=-T_{\mathbf{A}}^{a}$ for a real representation.

Specializing to $S U(N)$, there is another way to derive the fourth-order symmetric symbol. First, remember that,

$$
T_{\mathbf{F}}^{a} T_{\mathbf{F}}^{b}=\frac{1}{N} I_{2}(\mathbf{F}) \delta^{a b}+\frac{I_{3}(\mathbf{F})}{4 I_{2}(\mathbf{F})} d^{a b c} T_{\mathbf{F}}^{c}+\frac{i}{2} I_{2}(\mathbf{F}) f^{a b c} T_{\mathbf{F}}^{c}
$$

With this, we can derive

$$
\operatorname{Tr}\left[\left\{T_{\mathbf{F}}^{a} T_{\mathbf{F}}^{b}\right\}\left\{T_{\mathbf{F}}^{c} T_{\mathbf{F}}^{d}\right\}\right]=\frac{4 I_{2}(\mathbf{F})^{2}}{N} \delta^{a b} \delta^{c d}+\frac{I_{3}(\mathbf{F})^{2}}{4 I_{2}(\mathbf{F})} d^{a b e} d^{c d e}=\frac{1}{N} \delta^{a b} \delta^{c d}+\frac{1}{2} d^{a b e} d^{c d e} .
$$

On the other hand, this trace can be computed using the general reduction in terms of invariant, giving

$$
\operatorname{Tr}\left[\left\{T_{\mathbf{F}}^{a} T_{\mathbf{F}}^{b}\right\}\left\{T_{\mathbf{F}}^{c} T_{\mathbf{F}}^{d}\right\}\right]=4 I_{4}(\mathbf{F}) d^{a b c d}+\frac{1}{3} I_{2}(\mathbf{F})\left(f^{a c e} f^{b d e}+f^{a d e} f^{b c e}\right)+4 \Lambda(\mathbf{F})\left(\delta^{a b} \delta^{c d}+\delta^{a c} \delta^{b d}+\delta^{a d} \delta^{b c}\right) .
$$

Combining the two,

$I_{4}(\mathbf{F}) d^{a b c d}=\frac{I_{3}(\mathbf{F})^{2}}{16 I_{2}(\mathbf{F})} d^{a b e} d^{c d e}-\frac{I_{2}(\mathbf{F})}{12}\left(f^{a c e} f^{b d e}+f^{a d e} f^{b c e}\right)-\Lambda(\mathbf{F})\left(\delta^{a b} \delta^{c d}+\delta^{a c} \delta^{b d}+\delta^{a d} \delta^{b c}\right)+\frac{I_{2}(\mathbf{F})^{2}}{N} \delta^{a b} \delta^{c d}$.

With the convention $I_{4}(\mathbf{F})=1$, this identity permits us to compute the quartic symbol $d^{a b c d}$ directly out of the lower-rank invariants. We can now check that for $N=3, I_{2}(\mathbf{F})=1 / 2, I_{3}(\mathbf{F})=1, I_{4}(\mathbf{F})=0$ and $\Lambda(\mathbf{F})=1 / 24$,

$$
0=\frac{1}{8} d^{a b e} d^{c d e}-\frac{1}{24}\left(f^{a c e} f^{b d e}+f^{a d e} f^{b c e}\right)-\frac{1}{24}\left(\delta^{a c} \delta^{b d}-\delta^{a b} \delta^{c d}+\delta^{a d} \delta^{b c}\right),
$$

which gives back the identity in Eq. (24). For $N=2, I_{2}(\mathbf{F})=1 / 2, I_{3}(\mathbf{F})=I_{4}(\mathbf{F})=0, \Lambda(\mathbf{F})=1 / 24$, we recover the usual reduction formula for the Levi-Civita tensor:

$$
0=-\frac{1}{24}\left(\varepsilon^{a c e} \varepsilon^{b d e}+\varepsilon^{a d e} \varepsilon^{b c e}\right)-\frac{1}{24}\left(\delta^{a c} \delta^{b d}-2 \delta^{a b} \delta^{c d}+\delta^{a d} \delta^{b c}\right) .
$$

\section{Casimir invariants for simple groups}

Thanks to the orthogonality condition adopted to fix $\Lambda(\mathbf{R})$ [34], the usual formula can be employed to get the explicit values of the invariant $I_{4}(\mathbf{R})$ for various representations,

$$
\begin{gathered}
I_{n}(\mathbf{R})=(-1)^{n} I_{n}\left(\mathbf{R}^{\dagger}\right), \\
I_{n}\left(\mathbf{R}_{1} \oplus \mathbf{R}_{2}\right)=I_{n}\left(\mathbf{R}_{1}\right)+I_{n}\left(\mathbf{R}_{2}\right), \\
I_{n}\left(\mathbf{R}_{1} \otimes \mathbf{R}_{2}\right)=I_{n}\left(\mathbf{R}_{1}\right) N\left(\mathbf{R}_{2}\right)+I_{n}\left(\mathbf{R}_{2}\right) N\left(\mathbf{R}_{1}\right) \\
=\sum I_{n}\left(\mathbf{R}_{i}^{\prime}\right),
\end{gathered}
$$

with $n=2,3,4$ and where $\mathbf{R}_{1} \otimes \mathbf{R}_{2}=\sum_{i} \mathbf{R}_{i}^{\prime}$. Altogether, these relations are more than sufficient to derive the Casimir invariants for any of the standard Lie algebra.
We give in Tables V-VII their values for the first few representations of some unitary and orthogonal algebras of rank $r \leq 5$, along with $\Lambda(\mathbf{R})$. We also checked these numbers by computing $I_{2,3,4}(\mathbf{R})$ directly using explicit matrix representations for the first few representations of each algebra. These numbers are compatible with the explicit formula in terms of Dynkin indices given in Ref. [34], up to the normalization conventions.

The normalization of the generators adopted for $S O(N)$ algebras in Tables VI and VII is not standard but physically inspired. Specifically, the invariants of an algebra $M$ can be expressed in terms of that of its subalgebra $N$. For instance, if a representation $\mathbf{R}_{M}$ branches into the sum of representations $\mathbf{R}_{N}$, we have the simple sum rule:

$$
I_{n}\left(\mathbf{R}_{M}\right)=\eta \sum_{\mathbf{R}_{N} \subset \mathbf{R}_{M}} I_{n}\left(\mathbf{R}_{N}\right),
$$


TABLE V. First few representations of $S U(N), N=2,3,4,5$, labelled by their Dynkin index, and their dimensions, quadratic, cubic, and quartic Casimir invariants, together with $\Lambda(\mathbf{R})$ as given by Eq. (B6).

\begin{tabular}{|c|c|c|c|c|c|c|c|c|c|c|}
\hline \multicolumn{11}{|c|}{$S U(2)$} \\
\hline $\mathbf{R}$ & (1) & (2) & (3) & (4) & (5) & (6) & (7) & (8) & (9) & (10) \\
\hline$N$ & 2 & 3 & 4 & 5 & 6 & 7 & 8 & 9 & 10 & 11 \\
\hline$I_{2}$ & $1 / 2$ & 2 & 5 & 10 & $35 / 2$ & 28 & 42 & 60 & $165 / 2$ & 110 \\
\hline$\Lambda$ & $\frac{1}{24}$ & $\frac{2}{3}$ & $\frac{41}{12}$ & $\frac{34}{3}$ & $\frac{707}{24}$ & $\frac{196}{3}$ & $\frac{259}{2}$ & 236 & $\frac{3223}{8}$ & $\frac{1958}{3}$ \\
\hline \multicolumn{11}{|c|}{$S U(3)$} \\
\hline $\mathbf{R}$ & (10) & (20) & (11) & (30) & (21) & (40) & (05) & (13) & (22) & (60) \\
\hline$N$ & 3 & 6 & 8 & 10 & 15 & $15^{\prime}$ & 21 & 24 & 27 & 28 \\
\hline$I_{2}$ & $1 / 2$ & $5 / 2$ & 3 & $15 / 2$ & 10 & $35 / 2$ & 35 & 25 & 27 & 63 \\
\hline$I_{3}$ & 1 & 7 & 0 & 27 & 14 & 77 & -182 & -64 & 0 & 378 \\
\hline$\Lambda$ & $\frac{1}{24}$ & $\frac{17}{24}$ & $\frac{3}{4}$ & $\frac{33}{8}$ & $\frac{29}{6}$ & $\frac{371}{24}$ & $\frac{539}{12}$ & $\frac{235}{12}$ & $\frac{81}{4}$ & $\frac{441}{3}$ \\
\hline \multicolumn{11}{|c|}{$S U(4)$} \\
\hline $\mathbf{R}$ & (100) & (010) & $(200)$ & (101) & (011) & $(020)$ & $(003)$ & $(400)$ & (201) & (210) \\
\hline$N$ & 4 & 6 & 10 & 15 & 20 & $20^{\prime}$ & $20^{\prime \prime}$ & 35 & 36 & 45 \\
\hline$I_{2}$ & $1 / 2$ & 1 & 3 & 4 & $13 / 2$ & 8 & $21 / 2$ & 28 & $33 / 2$ & 24 \\
\hline$I_{3}$ & 1 & 0 & 8 & 0 & -7 & 0 & -35 & 112 & 21 & 48 \\
\hline$I_{4}$ & 1 & -4 & 12 & 8 & -11 & -56 & 69 & 272 & 57 & 24 \\
\hline$\Lambda$ & $\frac{29}{816}$ & $\frac{11}{102}$ & $\frac{23}{34}$ & $\frac{40}{51}$ & $\frac{1313}{816}$ & $\frac{128}{51}$ & $\frac{1211}{272}$ & $\frac{56}{2}$ & $\frac{1639}{272}$ & $\frac{176}{17}$ \\
\hline \multicolumn{11}{|c|}{$S U(5)$} \\
\hline $\mathbf{R}$ & (1000) & (0100) & (2000) & (1001) & (0003) & (0011) & (0101) & (0020) & (2001) & (0110) \\
\hline$N$ & 5 & 10 & 15 & 24 & 35 & 40 & 45 & 50 & 70 & 75 \\
\hline$I_{2}$ & $1 / 2$ & $3 / 2$ & $7 / 2$ & 5 & 14 & 11 & 12 & $35 / 2$ & $49 / 2$ & 25 \\
\hline$I_{3}$ & 1 & 1 & 9 & 0 & -44 & -16 & -6 & -15 & 29 & 0 \\
\hline$I_{4}$ & 1 & -3 & 13 & 10 & 82 & -2 & -6 & -55 & 79 & -70 \\
\hline$\Lambda$ & $\frac{47}{1560}$ & $\frac{83}{520}$ & $\frac{77}{120}$ & $\frac{125}{156}$ & $\frac{1841}{390}$ & $\frac{1903}{780}$ & $\frac{167}{65}$ & $\frac{1589}{312}$ & $\frac{11123}{1560}$ & $\frac{1075}{156}$ \\
\hline
\end{tabular}

TABLE VI. First few representations of $S O(N), N=5,7,9,10$, labelled by their Dynkin index, and their dimensions, quadratic, and quartic Casimir invariants, together with $\Lambda(\mathbf{R})$ as given by Eq. (B6). The cubic invariant vanishes for all these algebras. The normalizations of the generators and of the quartic symbols is fixed in terms of that adopted for $S U(N)$ algebras, using Eq. (B25). The $S O(4)$ and $S O(6)$ algebras are not included since they are isomorphic to $S U(2) \otimes S U(2)$ and $S U(4)$, respectively. Note that the normalizations does not necessarily match, with for example $I_{2}(S U(4))=I_{2}(S O(6))$ but $I_{4}(S U(4))=-2 I_{4}(S O(6))$.

\begin{tabular}{|c|c|c|c|c|c|c|c|c|c|c|}
\hline \multicolumn{11}{|c|}{$S O(5)$} \\
\hline $\mathbf{R}$ & (10) & (01) & $(02)$ & (20) & (11) & (03) & (30) & (12) & (04) & (21) \\
\hline$N$ & 5 & 4 & 10 & 14 & 16 & 20 & 30 & 35 & $35^{\prime}$ & 40 \\
\hline$I_{2}$ & 1 & $1 / 2$ & 3 & 7 & 6 & $21 / 2$ & 27 & 21 & 28 & 29 \\
\hline$I_{4}$ & 2 & $-1 / 2$ & -6 & 26 & 6 & $-69 / 2$ & 162 & -6 & -132 & 91 \\
\hline$\Lambda$ & $\frac{1}{8}$ & $\frac{1}{32}$ & $\frac{5}{8}$ & $\frac{21}{8}$ & $\frac{13}{8}$ & $\frac{133}{32}$ & $\frac{153}{8}$ & $\frac{77}{8}$ & $\frac{35}{2}$ & $\frac{261}{16}$ \\
\hline \multicolumn{11}{|c|}{$S O(7)$} \\
\hline $\mathbf{R}$ & (100) & (001) & $(010)$ & (200) & (002) & (101) & $(300)$ & (110) & (011) & (003) \\
\hline$N$ & 7 & 8 & 21 & 27 & 35 & 48 & 77 & 105 & 112 & 112 \\
\hline$I_{2}$ & 1 & 1 & 5 & 9 & 10 & 14 & 44 & 45 & 46 & 54 \\
\hline$I_{4}$ & 2 & -1 & -2 & 30 & -16 & 10 & 220 & 42 & -46 & -126 \\
\hline$\Lambda$ & $\frac{13}{138}$ & $\frac{43}{552}$ & $\frac{125}{138}$ & $\frac{111}{46}$ & $\frac{155}{69}$ & $\frac{889}{276}$ & $\frac{1474}{69}$ & $\frac{735}{46}$ & $\frac{187}{12}$ & $\frac{2007}{92}$ \\
\hline \multicolumn{11}{|c|}{$S O(9)$} \\
\hline $\mathbf{R}$ & (1000) & (0001) & $(0100)$ & (2000) & (0010) & (0002) & (1001) & (3000) & (1100) & (0101) \\
\hline$N$ & 9 & 16 & 36 & 44 & 84 & 126 & 128 & 156 & 231 & 432 \\
\hline$I_{2}$ & 1 & 2 & 7 & 11 & 21 & 35 & 32 & 65 & 77 & 150 \\
\hline$I_{4}$ & 2 & -2 & 2 & 34 & -18 & -50 & 16 & 286 & 106 & -54 \\
\hline$\Lambda$ & $\frac{17}{228}$ & $\frac{10}{57}$ & $\frac{245}{228}$ & $\frac{517}{228}$ & $\frac{329}{76}$ & $\frac{1855}{228}$ & $\frac{376}{57}$ & $\frac{5395}{228}$ & $\frac{5005}{228}$ & $\frac{850}{19}$ \\
\hline \multicolumn{11}{|c|}{$S O(10)$} \\
\hline $\mathbf{R}$ & $(10000)$ & (00001) & $(01000)$ & $(20000)$ & (00100) & (00002) & (10010) & (00011) & $(30000)$ & (11000) \\
\hline$N$ & 10 & 16 & 45 & 54 & 120 & 126 & 144 & 210 & $210^{\prime}$ & 320 \\
\hline$I_{2}$ & 1 & 2 & 8 & 12 & 28 & 35 & 34 & 56 & 77 & 96 \\
\hline$I_{4}$ & 2 & -2 & 4 & 36 & -16 & -50 & 14 & -68 & 322 & 144 \\
\hline$\Lambda$ & $\frac{19}{282}$ & $\frac{103}{564}$ & $\frac{160}{141}$ & $\frac{104}{47}$ & $\frac{770}{141}$ & $\frac{2345}{282}$ & $\frac{3791}{564}$ & $\frac{1792}{141}$ & $\frac{7007}{282}$ & $\frac{1168}{47}$ \\
\hline
\end{tabular}


TABLE VII. First few representations of $S O(8)$, labeled by their Dynkin index. Because of the invariance of the eight-dimensional Levi-Civita tensor, this algebra has a second quartic invariant tensor. Its normalization is fixed to make manifest the relationship between the values of both quartic Casimir invariants, and corresponds to $\eta=-1 / 8$ in Eq. (B9). A second feature of $S O(8)$ is its triality symmetry: dimensions and quadratic Casimir invariants are the same under permutations of the first, third, and fourth simple root. Both quartic Casimir invariants vanish when summed over representations linked by the permutation symmetry [34]. This means in particular that they vanish identically for the 28,300 , and 350 .

\begin{tabular}{|c|c|c|c|c|c|c|c|c|c|c|}
\hline \multicolumn{11}{|c|}{$S O(8)$} \\
\hline & \multicolumn{3}{|c|}{$8\left(I_{2}, \Lambda=1,1 / 12\right)$} & \multicolumn{3}{|c|}{$\mathbf{1 1 2}\left(I_{2}, \Lambda=54,45 / 2\right)$} & \multicolumn{3}{|c|}{$\mathbf{2 2 4}\left(I_{2}, \Lambda=100,115 / 3\right)$} & \multirow{2}{*}{$\begin{array}{c}\mathbf{2 8}\left(I_{2}, \Lambda=6,1\right) \\
(\mathbf{0 1 0 0})\end{array}$} \\
\hline & (1000) & $(0001)$ & (0010) & (2000) & (0002) & $(0020)$ & (1002) & (1020) & (2001) & \\
\hline$I_{4}$ & 2 & -1 & -1 & 252 & -126 & -126 & -40 & -40 & 212 & 0 \\
\hline \multirow[t]{3}{*}{$I_{4}^{\prime}$} & 0 & -1 & 1 & 0 & -126 & 126 & -128 & 128 & -44 & 0 \\
\hline & \multicolumn{3}{|c|}{$\mathbf{3 5}\left(I_{2}, \Lambda=10,7 / 3\right)$} & \multicolumn{3}{|c|}{$\mathbf{1 6 0}\left(I_{2}, \Lambda=60,19\right)$} & & & & $\mathbf{3 0 0}\left(I_{2}, \Lambda=150,65\right)$ \\
\hline & (2000) & $(0002)$ & $(0020)$ & (1100) & (0101) & (0110) & $(0012)$ & $(0021)$ & (2010) & $(0200)$ \\
\hline$I_{4}$ & 32 & -16 & -16 & 72 & -36 & -36 & -172 & -172 & 212 & 0 \\
\hline$I_{4}^{\prime}$ & 0 & -16 & 16 & 0 & -36 & 36 & -84 & 84 & 44 & 0 \\
\hline & \multicolumn{3}{|c|}{$\mathbf{5 6}\left(I_{2}, \Lambda=15,13 / 4\right)$} & \multicolumn{3}{|c|}{$\mathbf{2 9 4}\left(I_{2}, \Lambda=210,133\right)$} & \multicolumn{3}{|c|}{$\mathbf{5 6 7}\left(I_{2}, \Lambda=324,162\right)$} & $\mathbf{3 5 0}\left(I_{2}, \Lambda=150,55\right)$ \\
\hline & (0011) & (1001) & (1010) & (4000) & (0004) & $(0040)$ & (2100) & $(0102)$ & $(0120)$ & $(1011)$ \\
\hline$I_{4}$ & -18 & 9 & 9 & 1344 & -672 & -672 & 864 & -432 & -432 & 0 \\
\hline$I_{4}^{\prime}$ & 0 & 9 & -9 & 0 & -672 & 672 & 0 & -432 & 432 & 0 \\
\hline
\end{tabular}

where $\eta$ is a constant reflecting the normalization convention adopted for the generators of $M$ and $N$. In Table VI, we chose to fix $\eta=1$. For example, the generators in the defining representation of $S O(10)$ are normalized so that

$$
I_{2}(\mathbf{1 0})^{S O(10)}=I_{2}(\overline{\mathbf{5}})^{S U(5)}+I_{2}(\mathbf{5})^{S U(5)}=1,
$$

since $\mathbf{1 0} \rightarrow \overline{\mathbf{5}}+\mathbf{5}$. Similarly, the normalization of the quartic symbol of $S O(10)$ is then fixed by imposing $I_{4}(\mathbf{1 0})^{S O(10)}=2 I_{4}(\mathbf{5})^{S U(5)}=2$. This makes sense physically if one thinks of a field in a given $S O(10)$ representation circulating in some loop. Our normalization conventions make the matching of this amplitude to that computed in terms of the fields of the subalgebra most transparent. Note that the generators and quartic symbols of all $S O(N)$ algebras are fixed once that of $S O(10)$ is, since $S O(N) \subset S O(N+1)$. Further, we also checked that these conventions are compatible with $S O(3) \otimes S O(7) \subset S O(10)$ and $S O(4) \otimes S O(6) \subset S O(10)$, with $S O(4) \sim S U(2) \otimes S U(2)$.

Other relations between the invariants of an algebra and that of its subalgebras are given in the text, see in particular Eq. (53) which gives $I_{n}\left(\mathbf{R}_{M}\right)$ in terms of $I_{n-1}\left(\mathbf{R}_{N}\right)$, Eq. (53) which fixes $I_{4}\left(\mathbf{R}_{M}\right)$ in terms of $I_{2}\left(\mathbf{R}_{N}\right)$, or Eq. (48) which gives $I_{n}\left(\mathbf{R}_{M}\right)$ in terms of the $U(1)$ charges of the $\mathbf{R}_{M}$ states. To close this section, let us give a few illustrations for these relations.

Consider first the reduction of $S U(2)$ down to the $U(1)$ subgroup of $S U(2)$ generated by $T^{3}$. Since there is no quartic invariant for $S U(2)$, Eq. (48) is easy to check. The fundamental $S U(2)$ representation corresponds to two complex states of charge $\left|T^{3}\right|=1 / 2$, so we can identify $2(1 / 2)^{4}=3 \Lambda^{S U(2)}(\mathbf{2})$ since $\Lambda^{S U(2)}(\mathbf{2})=1 / 24$. Similarly, the complex adjoint representation of $S U(2)$ contains two states of unit charge, hence $2=3 \Lambda^{S U(2)}(\mathbf{3})$, and the isospin $3 / 2$ decomposes into four states such that $2\left((1 / 2)^{4}+(3 / 2)^{4}\right)=3 \Lambda^{S U(2)}(4)$, which give back the correct values $\Lambda^{S U(2)}(\mathbf{3})=2 / 3$ and $\Lambda^{S U(2)}(\mathbf{4})=41 / 12$. The same exercise can be repeated for $S U(3)$, for which the absence of the quartic invariant ensures that $\operatorname{Tr}\left(\left(T_{\mathbf{R}}^{3}\right)^{4}\right)=$ $\operatorname{Tr}\left(\left(T_{\mathbf{R}}^{8}\right)^{4}\right)$ if $T^{3}$ and $T^{8}$ are the conventional Cartan generators (equal to half the corresponding Gell-Mann matrices in the fundamental representation). To apply the same method for $S U(5)$, we need to first fix two free parameters. Specifically, we start from

$$
3 \Lambda(\mathbf{R})+d^{\alpha \alpha \alpha \alpha} I_{4}(\mathbf{R})=\delta \sum_{q_{\alpha} \in \mathbf{R}} q_{\alpha}^{4},
$$

The value of $d^{\alpha \alpha \alpha \alpha}$ and the $U(1)$ generator normalization $\delta$ [which was coincidentally equal to one in the previous $S U(2)$ example] need to be fixed. If we identify $T^{\alpha}$ as the hypercharge generator in the subalgebra $S U(3) \otimes S U(2) \otimes U(1) \subset S U(5)$, we can use the branching rules [60]

$$
\begin{aligned}
\mathbf{5} & =(\mathbf{3}, \mathbf{1})_{2}+(\mathbf{1}, \mathbf{2})_{-3}, \\
\mathbf{1 0} & =(\overline{\mathbf{3}}, \mathbf{1})_{4}+(\mathbf{3}, \mathbf{2})_{-1}+(\mathbf{1}, \mathbf{1})_{-6},
\end{aligned}
$$

and these two constants are fixed as 


$$
\begin{aligned}
& \left\{\begin{array}{l}
I_{4}(\mathbf{5}) d^{\alpha \alpha \alpha \alpha}+3 \Lambda(\mathbf{5})=\delta\left(2 \times 3^{4}+3 \times 2^{4}\right) \\
I_{4}(\mathbf{1 0}) d^{\alpha \alpha \alpha \alpha}+3 \Lambda(\mathbf{1 0})=\delta\left(6^{4}+3 \times 4^{4}+3 \times 2 \times 1^{4}\right)
\end{array}\right. \\
& \rightarrow\left\{\begin{array}{l}
\delta=1 / 60^{2} \\
d^{\alpha \alpha \alpha \alpha}=-5 / 156 .
\end{array}\right.
\end{aligned}
$$

One can then check that the $I_{4}$ for the other $S U(5)$ representations are correctly reproduced.

The same branching rules can be used in connection with Eq. (53), which we write as

$$
I_{4}\left(\mathbf{R}_{M}\right)=\eta \sum_{\mathbf{R}_{N} \subset \mathbf{R}_{M}} q_{\alpha}\left(\mathbf{R}_{N}\right) I_{3}\left(\mathbf{R}_{N}\right)
$$

The subalgebra needs not be maximal so we consider $U(1) \otimes S U(3) \subset S U(5)$. Using the values quoted in Table V, the first rule of Eq. (B28) translates into $I_{4}^{S U(5)}(\mathbf{5})=2 \eta I_{3}^{S U(3)}(\mathbf{3})$ and fixes $\eta=1 / 2$. Then, we can check that this equation is valid for all the other $S U(5)$ representations listed in Table V.
[1] W. Heisenberg and H. Euler, Z. Phys. 98, 714 (1936).

[2] E. Fermi, Nuovo Cimento 11, 1 (1934).

[3] S. Weinberg, Physica Amsterdam) 96A, 327 (1979); J. Gasser and H. Leutwyler, Ann. Phys. (N.Y.) 158, 142 (1984); R. Machleidt and D. R. Entem, Phys. Rep. 503, 1 (2011).

[4] S. Weinberg, Proc. Sci., CD09 (2009) 001.

[5] H. Georgi, Annu. Rev. Nucl. Part. Sci. 43, 209 (1993).

[6] S. Weinberg, Phys. Rev. Lett. 43, 1566 (1979).

[7] D. B. Kaplan, Nucl. Phys. A663, 155 (2000).

[8] M. Neubert, arXiv:hep-ph/0512222.

[9] W. Skiba, arXiv:1006.2142.

[10] S. Fichet and G. von Gersdorff, J. High Energy Phys. 03 (2014) 102.

[11] D. de Florian et al. (LHC Higgs Cross Section Working Group), arXiv:1610.07922.

[12] W. Buchmuller and D. Wyler, Nucl. Phys. B268, 621 (1986); B. Grzadkowski, M. Iskrzynski, M. Misiak, and J. Rosiek, J. High Energy Phys. 10 (2010) 085.

[13] A. De Simone and T. Jacques, Eur. Phys. J. C 76, 367 (2016).

[14] C. Cheung, P. Creminelli, A. L. Fitzpatrick, J. Kaplan, and L. Senatore, J. High Energy Phys. 03 (2008) 014.

[15] J. J. M. Carrasco, M. P. Hertzberg, and L. Senatore, J. High Energy Phys. 09 (2012) 082.

[16] A. Cadene, P. Berceau, M. Fouche, R. Battesti, and C. Rizzo, Eur. Phys. J. D 68, 16 (2014).

[17] F. D. Valle, A. Ejlli, U. Gastaldi, G. Messineo, E. Milotti, R. Pengo, G. Ruoso, and G. Zavattini, Eur. Phys. J. C 76, 24 (2016).

[18] T. Yamazaki, T. Inada, T. Namba, S. Asai, T. Kobayashi, A. Matsuo, K. Kindo, and H. Nojiri, Nucl. Instrum. Methods A 833, 122 (2016).

[19] R. P. Mignani, V. Testa, D. G. Caniulef, R. Taverna, R. Turolla, S. Zane, and K. Wu, Mon. Not. R. Astron. Soc. 465, 492 (2017).

[20] L. M. Capparelli, A. Damiano, L. Maiani, and A. D. Polosa, Eur. Phys. J. C 77, 754 (2017).

[21] M. Born and L. Infeld, Proc. R. Soc. A 144, 425 (1934); 147, 522 (1934).

[22] A. A. Tseytlin, in The Many Faces of the Superworld, edited by M. A. Shifman, (World Scientific, Singapore, 2000), pp. 417-452.
[23] J. Ellis, N. E. Mavromatos, and T. You, Phys. Rev. Lett. 118, 261802 (2017).

[24] J. Ellis and S. F. Ge, Phys. Rev. Lett. 121, 041801 (2018).

[25] J. A. Gracey, Nucl. Phys. B634, 192 (2002); 696, 295(E) (2004); Phys. Rev. D 97, 025009 (2018).

[26] M. K. Gaillard, Nucl. Phys. B268, 669 (1986).

[27] O. Cheyette, Nucl. Phys. B297, 183 (1988).

[28] B. Henning, X. Lu, and H. Murayama, J. High Energy Phys. 01 (2016) 023.

[29] A. Drozd, J. Ellis, J. Quevillon, and T. You, J. High Energy Phys. 03 (2016) 180.

[30] S. A. R. Ellis, J. Quevillon, T. You, and Z. Zhang, Phys. Lett. B 762, 166 (2016).

[31] S. A. R. Ellis, J. Quevillon, T. You, and Z. Zhang, J. High Energy Phys. 08 (2017) 054.

[32] Z. Zhang, J. High Energy Phys. 05 (2017) 152.

[33] F. Boudjema, Phys. Lett. B 187, 362 (1987).

[34] S. Okubo, J. Math. Phys. (N.Y.) 23, 8 (1982).

[35] T. van Ritbergen, A. N. Schellekens, and J. A. M. Vermaseren, Int. J. Mod. Phys. A 14, 41 (1999).

[36] For an introduction, see e.g., A. Dobado, A. Gomez-Nicola, A. L. Maroto, and J. R. Pelaez, Effective lagrangians for the standard model (Springer-Verlag, New York, 1997).

[37] T. Appelquist and J. Carazzone, Phys. Rev. D 11, 2856 (1975).

[38] D. V. Vassilevich, Phys. Rep. 388, 279 (2003).

[39] A. E. M. van de Ven, Classical Quantum Gravity 15, 2311 (1998).

[40] L. L. Salcedo, Eur. Phys. J. C 37, 511 (2004).

[41] A. Drozd, J. Ellis, J. Quevillon, and T. You, J. High Energy Phys. 06 (2015) 028.

[42] M. Reuter, M. G. Schmidt, and C. Schubert, Ann. Phys. (N.Y.) 259, 313 (1997).

[43] D. Fliegner, P. Haberl, M. G. Schmidt, and C. Schubert, Ann. Phys. (N.Y.) 264, 51 (1998).

[44] N. Ahmadiniaz and C. Schubert, AIP Conf. Proc. 1492, 199 (2012).

[45] T. Hahn, Comput. Phys. Commun. 140, 418 (2001).

[46] R. Mertig, M. Bohm, and A. Denner, Comput. Phys. Commun. 64, 345 (1991); V. Shtabovenko, R. Mertig, and F. Orellana, Comput. Phys. Commun. 207, 432 (2016).

[47] H. H. Patel, Comput. Phys. Commun. 197, 276 (2015).

[48] V. Shtabovenko, Comput. Phys. Commun. 218, 48 (2017). 
[49] W. H. Furry, Phys. Rev. 51, 125 (1937).

[50] E. A. Uehling, Phys. Rev. 48, 55 (1935).

[51] H. Fanchiotti, H. O. Girotti, and A. Sirlin, Lett. Nuovo Cimento 4, 826 (1972); 4, 826 (1972).

[52] F. X. Dong, X. D. Jiang, and X. J. Zhou, J. Phys. G 19, 969 (1993).

[53] S. Weinberg, in Lectures on Elementary Particles and Quantum Field Theory, edited by S. Deser, M. Grisaru, and H. Pendleton (MIT Press, Cambridge, MA, 1970), Vol. 1.

[54] F. Preucil and J. Horejší, J. Phys. G 45, 085005 (2018).

[55] J. Pasukonis, arXiv:0710.0159.

[56] M. Bace and N. D. H. Dass, Ann. Phys. (N.Y.) 94, 349 (1975).

[57] M. B. Gavela, G. Girardi, C. Malleville, and P. Sorba, Nucl. Phys. B193, 257 (1981).
[58] G. Belanger, F. Boudjema, J. Fujimoto, T. Ishikawa, T. Kaneko, K. Kato, and Y. Shimizu, Phys. Rep. 430, 117 (2006).

[59] E. H. Simmons, Phys. Lett. B 226, 132 (1989); 246, 471 (1990).

[60] R. Slansky, Phys. Rep. 79, 1 (1981).

[61] M. L. Laursen, K. O. Mikaelian, and M. A. Samuel, Phys. Rev. D 23, 2795 (1981).

[62] G. Belanger and F. Boudjema, Phys. Lett. B 288, 210 (1992); G. Belanger, F. Boudjema, Y. Kurihara, D. PerretGallix, and A. Semenov, Eur. Phys. J. C 13, 283 (2000).

[63] M. L. Laursen, M. A. Samuel, G. B. Tupper, and A. Sen, Phys. Rev. D 27, 196 (1983).

[64] M. Stohr and J. Horejsi, Phys. Rev. D 49, 3775 (1994); M. Baillargeon, F. Boudjema, E. Chopin, and V. Lafage, Z. Phys. C 71, 431 (1996). 